Article

\title{
A Novel Change Detection Approach for Multi-Temporal High-Resolution Remote Sensing Images Based on Rotation Forest and Coarse-to-Fine Uncertainty Analyses
}

\author{
Wenqing Feng ${ }^{1}(\mathbb{D})$, Haigang Sui ${ }^{1, *}$, Jihui Tu ${ }^{2}$, Weiming Huang ${ }^{3}{ }^{(D)}$, Chuan $X u^{1}$ \\ and Kaimin Sun ${ }^{1}$ (iD \\ 1 State Key Laboratory of Information Engineering in Surveying Mapping and Remote Sensing, \\ Wuhan University, Wuhan 430079, China; wq_feng@whu.edu.cn (W.F.); chuanxu@whu.edu.cn (C.X.); \\ sunkm@whu.edu.cn (K.S.) \\ 2 Electronics \& Information School of Yangtze University, Yangtze University, Jingzhou 434023, China; \\ tujh@yangtzeu.edu.cn \\ 3 Department of Physical Geography and Ecosystem Science, Lund University, 22362 Lund, Sweden; \\ weiming.huang@nateko.lu.se \\ * Correspondence: haigang_sui@263.net; Tel.: +86-27-6877-8876
}

Received: 13 May 2018; Accepted: 20 June 2018; Published: 25 June 2018

\begin{abstract}
In the process of object-based change detection (OBCD), scale is a significant factor related to extraction and analyses of subsequent change data. To address this problem, this paper describes an object-based approach to urban area change detection (CD) using rotation forest (RoF) and coarse-to-fine uncertainty analyses of multi-temporal high-resolution remote sensing images. First, highly homogeneous objects with consistent spatial positions are identified through vector-raster integration and multi-scale fine segmentation. The multi-temporal images are stacked and segmented under the constraints of a historical land use vector map using a series of optimal segmentation scales, ranging from coarse to fine. Second, neighborhood correlation image analyses are performed to highlight pixels with high probabilities of being changed or unchanged, which can be used as a prerequisite for object-based analyses. Third, based on the coarse-to-fine segmentation and pixel-based pre-classification results, change possibilities are calculated for various objects. Furthermore, changed and unchanged objects identified at different scales are automatically selected to serve as training samples. The spectral and texture features of each object are extracted. Finally, uncertain objects are classified using the RoF classifier. Multi-scale classification results are combined using a majority voting rule to generate the final $C D$ results. In experiments using two pairs of real high-resolution remote sensing datasets, our proposed approach outperformed existing methods in terms of $C D$ accuracy, verifying its feasibility and effectiveness.
\end{abstract}

Keywords: change detection; segmentation; scale; features; neighborhood correlation image analyses; rotation forest; majority voting

\section{Introduction}

Change detection (CD) is an important research topic involving quantitative analyses of multi-temporal remotely sensed images to investigate changes in land cover, particularly in the contexts of urban infrastructure monitoring, urban development, and disaster assessment [1]. Along with the rapid development of remotely sensed image acquisition systems and gradual shortening of the 
acquisition cycle, the application of $\mathrm{CD}$ is becoming increasingly widespread, and demand for such analyses is expanding. This demand increases the requirements and challenges for CD technology.

Over the past few years, a number of methods and effective models have been proposed [2-10]. Pixel-based change detection (PBCD) methods have been widely used in low- and medium-resolution images [2]. These traditional algorithms utilize a single pixel as the analyses unit and are unable to incorporate spatial relationships with neighboring pixels, leading to isolation and the "salt and pepper" phenomenon [1,2]. To overcome this problem, some scholars have employed the level set method and its improved algorithm [3,4], Markov Random Field (MRF) [5,6], Conditional Random Field (CRF) $[7,8]$, and other methods $[9,10]$, all of which reduce the uncertainty of CD by effectively combining spectral and spatial information. However, the quality of the control parameters for those methods seriously affects the accuracy of the final result. Parameter setting reduces the universality of those methods. If the spatial neighborhood relationship is not defined accurately enough, a smooth transition of edge details may occur, resulting in false detections or missed detections.

With improved resolution, the internal spectral difference between a particular pair of similar features gradually increases. Automated CD technology based on pixel spectral statistics cannot satisfactorily extract change information, and this problem is the main obstacle to widespread application of high-resolution remotely sensed images. The emergence of object-oriented technology for high-resolution remote sensing image analyses provides a new paradigm in that the basic unit of $\mathrm{CD}$ is changed from pixel to object [11]. Because the object-based change detection (OBCD) approach offers great advantages over the PBCD approach, it has received extensive attention and has been developed in recent years [12-17]. An object is defined as a single homogeneous region with similar spatial and spectral properties. Each object has characteristic features, such as its spectrum, shape, texture, and context. We can take full advantage of spectral features in combination with other features to improve accuracy of the CD process. To this end, the most commonly used methods are object-based (OB) change vector analyses (OCVA), OB correlation coefficient (OCC), and OB chi-square transformation (OCST) [18-22]. These methods perform a direct comparison of the two multispectral images under consideration. These unsupervised techniques leverage various features of the object, and incorporate them into the analyses at a later stage. These methods are suitable for some applications, such as the detection of deforestation or land-cover changes. The performance of these methods, however, relies heavily on the quality of feature selection, the allocation of feature weights, and the determination of the change threshold. Moreover, due to the difficulty of determining the segmentation scale, these methods are likely to introduce uncertainty into the CD process, thus reducing the reliability of the results. Although these methods can have good results, they are usually focused on one scale, which may not work well in more heterogeneous areas [23]. Because the landscape usually consists of different land cover types of varying size, it may not be possible to properly segment all features in a scene using a single scale [23]. To achieve better results, the segmentation scale, feature extraction, the change threshold, and many other factors must be considered.

Development of the OBCD method has made tremendous progress to date. However, the results rely heavily on the determination of the segmentation scale [24]. As objects of interest exist within a scene, often within a range of different sizes, shapes and times, no single spatial resolution is sufficient to capture all their characteristics [24]. Methods to objectively evaluate segmentation results, obtain the optimal segmentation scale, and avoid the influence of visual interpretation are increasingly important. Determination of the optimal segmentation scale is directly related to subsequent extraction and analyses of change information [25]. At present, the scale parameters can only be selected based on visual interpretation or evaluation of multi-scale segmentation results, i.e., using post-segmentation evaluation to optimize scale selection $[12,14,15]$. With these methods, multi-scale determination is based on experience or repeated experiments. Scale selection is usually based on visual interpretation, which is inevitably subjective. Because the scope of visual observation is limited, it is difficult to obtain optimal segmentation results. In addition, some researchers have 
utilized existing geographic information system (GIS) databases and prior knowledge to accomplish high-resolution remote sensing image CD [26-30]. Post-classification with multi-temporal remote sensing images is one of the most popular change detection methods, providing the detailed "from-to" change information in real applications [31,32]. However, due to the fact that it neglects the temporal correlation between corresponding pixels in multi-temporal images, the post-classification approach usually suffers from an accumulation of misclassification errors [32]. In order to solve this problem, some researchers have combined historical land use vector maps (HVMs) with remote sensing images to create a classification system, set decision rules, and used existing GIS knowledge to aid the CD process $[26,28-30]$. These studies use GIS and remote sensing tools to monitor land use and land cover changes at different spatiotemporal scales. The GIS and remote sensing tools can provide scientific procedures to analyze the pattern, rate, and trend of environmental change at all scales. Generally speaking, there are two types of change that can affect polygons in HVMs, global change and local change. Global changes are changes to the overall category of the target polygon (e.g., region B from impervious surface to building, and region $C$ from farmland to impervious surface in Figure $1 a, b$ ), while local changes are those within a target polygon's coverage (e.g., part of region A from bare land to impervious surface, and part of region D from settlement to impervious surface Figure 1a,b). To effectively detect areas where local changes have occurred in an HVM, a remote sensing image needs to be segmented under the constraints of the HVM to separate the area exhibiting a local change. Vector-raster integration methods avoid the manual setting of parameters for segmentation partition and each object is the smallest unit of the land use CD. Nonetheless, these methods have several drawbacks. First, HVMs and images must have strict geometric registration, because the registration error can affect the CD result. Second, an HVM is produced according to the national census geography standard of the country being analyzed. It often reflects land use based on classification criteria, while remote sensing images reflect the actual land coverage. Transformation is necessary before integrating these two data sources. Third, objects acquired through HVM are usually heterogeneous, and the classification of these objects is generally complex. These flaws are likely to cause poor CD results.

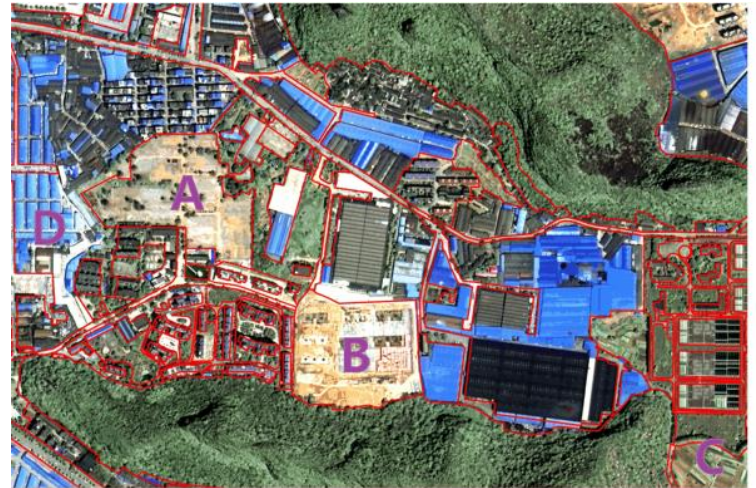

(a)

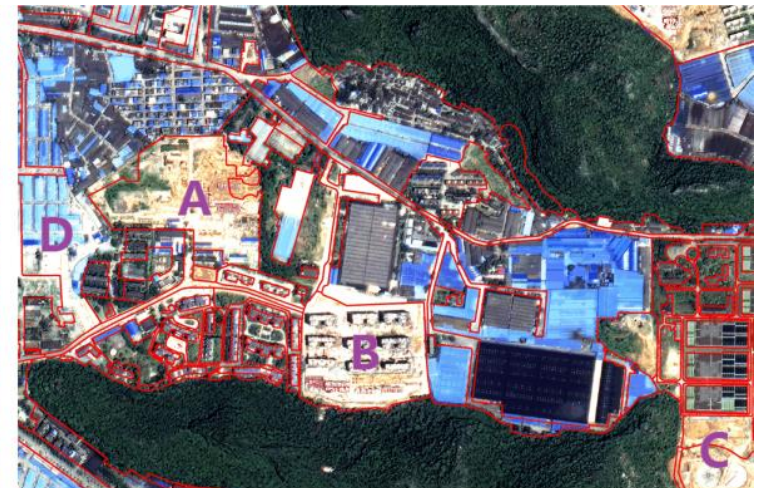

(b)

Figure 1. Examples of global and local change with regard to the polygons in an HVM. (a) T1 image overlaid with an HVM; (b) T2 image overlaid with an HVM.

To alleviate the aforementioned drawbacks, this paper incorporates the guidance of historical interpretation into the $\mathrm{OBCD}$ process from multi-temporal high-resolution remote sensing images. Accordingly, an $\mathrm{OBCD}$ approach that combines pixel-based pre-classification of high-resolution remote sensing images using the rotation forest (RoF) classifier and HVM is proposed in this paper. The purpose of combining two approaches is to obtain better results [16,33-35]. In general, there are two ways to undertake a combined approach: The two methods may be executed in parallel and then integrated, or the two methods may be performed consecutively, allowing the results of one 
method to be used as the premise for the other. Both of these strategies are employed for the purpose of achieving better results.

In this paper, we perform pixel-based and $\mathrm{OB}$ methods consecutively. For pixel-level analyses, neighborhood correlation image analysis (NCIA) is adopted to obtain pixel-level pre-classification results, which are then used as a prerequisite for OB analyses. The HVM is used as a priori knowledge to guide selection of the optimal segmentation scales for various ground objects. RoF, a new type of machine-learning algorithm, performs better than many single predictors and integrated forecasting methods due to its high diversity of training samples and features. RoF is widely used to combine numerous weak classifiers to generate a relatively strong classifier by reducing either the bias or the variance of the individual classifiers [23,36-38]. In this paper, we combine pixel-based and OB methods with the RoF model to analyze the influences of segmentation scale, sample selection, and feature extraction on final results. The main contributions of this paper are as follows. First, we propose a pre-classification scheme based on NCIA to obtain high-accuracy labeled samples. Second, we present a coarse-to-fine uncertainty analysis model based on the combination of RoF and HVM. In this analysis, the optimal image segmentation result is obtained under the guidance of historical knowledge interpretation. RoF, combining feature extraction and classifier ensembles, is applied to binary classification. Third, an effective majority voting (MV) rule is proposed to produce the final results.

The rest of this paper is organized as follows. Section 2 details the proposed method. In Section 3 , the experimental results on multiple datasets are presented to show the performance of the proposed method. Section 4 provides the discussion. Finally, we conclude the paper in Section 5.

\section{Methodology}

Among CD techniques, OBCD methods have become the most popular. However, due to the decline of spectral separability and complications such as identifying the same spectrum in different objects and/or different spectra in the same object, OBCD remains a challenging issue for several reasons. First, multi-temporal image segmentation (MTIS) is the foundation and basis of OBCD, and it seriously restricts the accuracy of OBCD. Not only do many segmentation algorithms exist that affect the resulting object geometries, but many CD techniques can affect the final results [24]. Second, change information is usually extracted at a single scale, neglecting scale set constraints. Third, objects in MTIS results are treated independently, ignoring the effects of neighboring object interactions on the change property.

To address these key problems, a multi-scale and multi-level CD strategy is adopted in this paper. The overall workflow of the proposed approach is shown in Figure 2. Four aspects are investigated to improve OBCD accuracy in terms of the OBCD process, namely MTIS, training sample selection, multi-feature extraction, and coarse-to-fine uncertainty analyses. (1) MTIS is conducted using vector-raster integration and coarse-to-fine segmentation. The superimposed image is divided into homogeneous objects with eCognition software (version 8.7) [39], facilitating subsequent CD experiments. (2) Changed and unchanged objects are selected based on coarse-to-fine segmentation and pixel-level pre-classification. Uncertain objects are further classified using the trained RoF model. (3) The multi-features of each object in the images are represented by spectral and texture information of homogeneous pixels. (4) Scale sets can be considered as a collection of image sequences of different scales. CD results from different scales are usually treated independently, and thus multi-scale fusion is implemented to combine different $C D$ results using coarse-to-fine uncertainty analyses. 


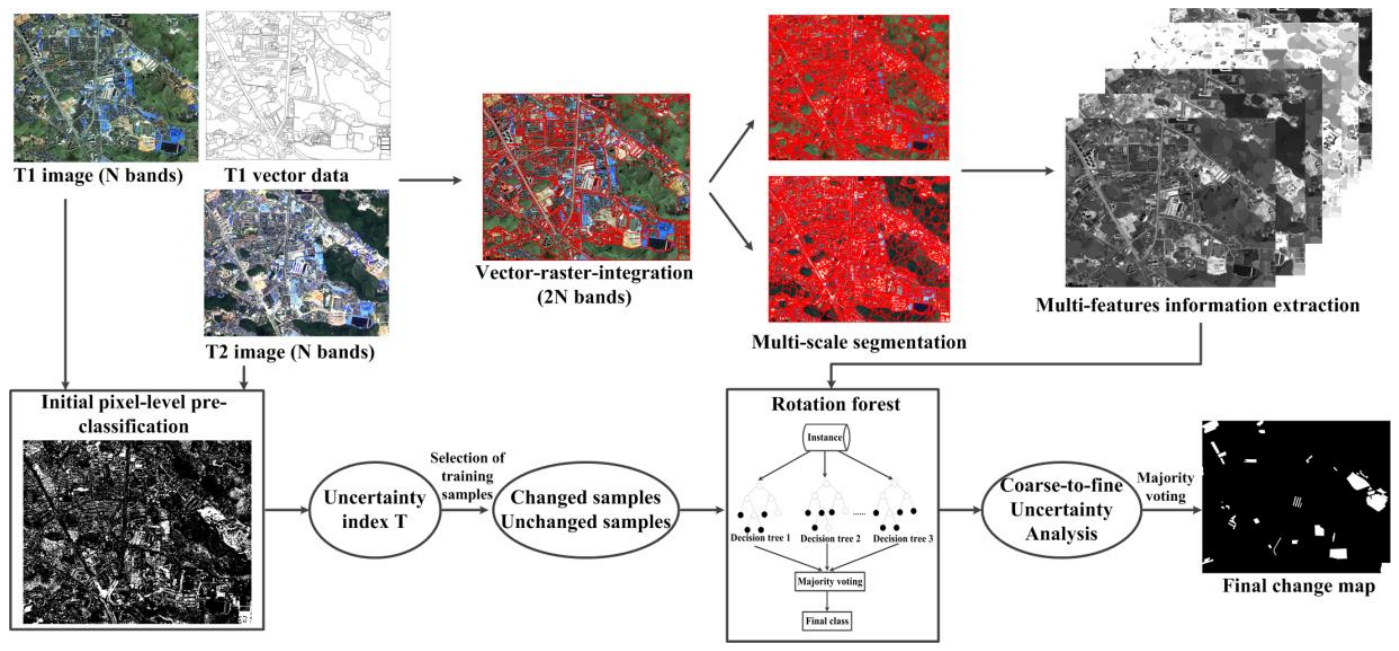

Figure 2. Flowchart of the proposed approach.

\subsection{MTIS and Estimation of Scale Parameters}

Multi-resolution segmentation (MRS) [39], which is embedded in eCognition software, is employed for image segmentation. Acquisition of coarse-to-fine sequential images is the basis of scale-driven OBCD. The quality of segmentation seriously affects the accuracy of object feature extraction and $\mathrm{OBCD}$. Based on different image segmentation strategies, there are three primary modes of multi-temporal image segmentation at present [40,41], namely single-temporal segmentation (STS), multi-temporal separate segmentation (MTSS), and multi-temporal combined segmentation (MTCS), as shown in Figure 3. Further details of the three primary modes of multi-temporal image segmentation are described in Niemeyer et al. (2008) and Zhang et al. (2017). In this paper, corresponding objects are extracted via vector-raster integration and coarse-to-fine segmentation under the boundary constraints of HVM. The HVM, produced from the National Census Geographic of China, is used as auxiliary data. The superimposed image is divided into homogeneous objects with consistent spatial positions.

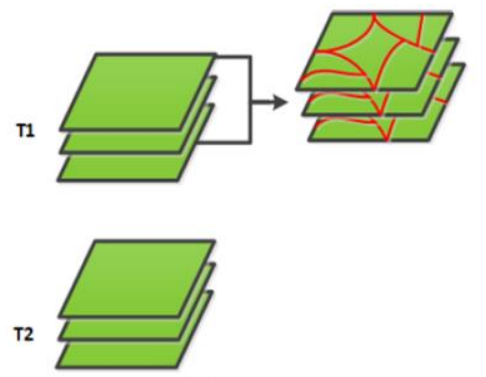

(a)

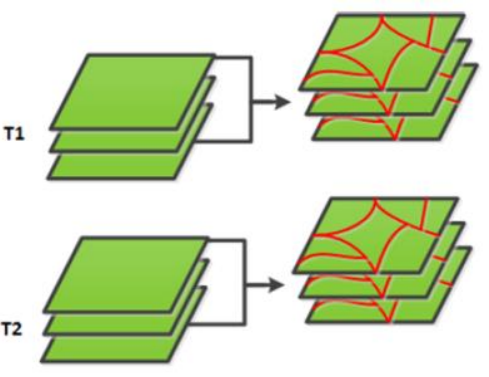

(b)

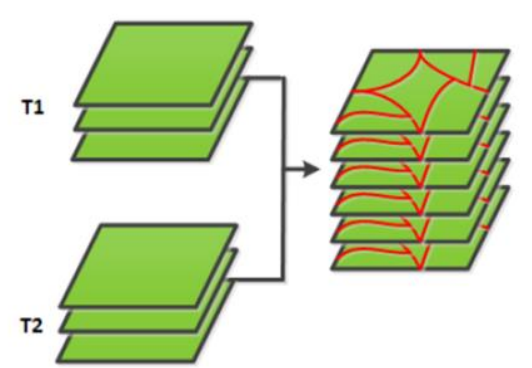

(c)

Figure 3. Multi-temporal image segmentation modes. (a) STS; (b) MTSS; (c) MTCS.

Estimation of scale parameter (ESP) [42,43] and the modified average segmentation evaluation index $(A S E I)$ are used to guide the selection of the optimal segmentation scales for different ground objects. ESP, produced by Lucian et al. (2010), is a scale parameter estimation plug-in developed for eCognition software that calculates the local variance (LV) of homogeneous image objects using different scale parameters. The average standard deviation of the object layer is used to determine whether the segmentation scale is optimal. The rate of change in LV (ROC-LV) is used to indicate the approximate optimal scale parameter. When the ROC-LV is at its maximum value within an interval, the corresponding scale is the optimal scale. 
However, this method can only be adapted to analyses of image data in a single band. To take full advantage of multi-spectral information, we utilize the modified ASEI to confirm the best scale parameter. The segmentation evaluation index (SEI) [44,45] in band $L$ for an object is defined by Equation (1), where $\sigma_{L}$ is the spectral standard deviation of the object, which is used as a homogeneity index. Its formula is shown in Equation (2), where $\Delta C_{L}$ is the heterogeneity index derived from calculating the absolute value of the mean difference between the object and a neighbouring object. Its formula is shown in Equation (3), in which num is the total number of pixels within the object, $C_{L i}$ is the grey value of pixel $i$ in band $L, \overline{C_{L}}$ is the mean value of the object, $l$ is the boundary length of the object, $l_{k}$ is the boundary length of the common edge with the $k$ th adjacent object, and $\overline{C_{L k}}$ is the mean value of the $k$ th adjacent object:

$$
\begin{gathered}
S E I_{L}=\frac{\Delta C_{L}}{\sigma_{L}} \\
\sigma_{L}=\sqrt{\frac{1}{n u m-1} \sum_{i=1}^{n u m}\left(C_{L i}-\overline{C_{L}}\right)^{2}} \\
\Delta C_{L}=\frac{1}{l} \sum_{k=1}^{K} l_{k}\left|\overline{C_{L}}-\overline{C_{L k}}\right|
\end{gathered}
$$

All of the formulas above are for single-band $L$ only. For superimposed images (number of bands is $2 N$, where $N$ is the band number of the single-phase image), eCognition applies different weights $\left(w_{L}\right)$ to different bands to highlight the impacts of various bands on the segmentation results. Therefore, when considering a plurality of the bands for the SEI of any object, this paper modifies the calculation formula of SEI to Equation (4), and uses a weight for each band of $w_{L}=1$. This ensures that each band is equal in the segmentation process [42,43]:

$$
S E I=\sum_{L=1}^{2 N} w_{L} \times S E I_{L}
$$

To compare objects at different segmentation scales, the SEI values of all objects in the study area can be averaged. Because the area (i.e., the number of pixels forming an image object) of the object may affect the segmentation results, this paper introduces the area factor, and gives a larger weight value to objects with larger areas to reduce the instability caused by object area. The modified ASEI is calculated using Equation (5):

$$
A S E I=\frac{1}{A} \sum_{i=1}^{N u m} A_{j} \times S E I_{j}
$$

where $A$ represents the total area of all objects, $A_{j}$ is the area of the $j$-th object, Num is the total number of objects, and $S E I_{j}$ is the $S E I$ value of the $j$-th object.

\subsection{Selection of Training Samples}

Neighbourhood correlation image analysis (NCIA) is a pixel-based method of CD, which is based on spectral contextual information created using correlation analyses between bi-temporal images within a specified neighborhood [46,47]. Further details of the NCIA method are described in Im et al. (2005). A rectangular (e.g., a moving window of $5 \times 5$ pixels) neighborhood type is used in this paper. After obtaining the difference image, the Otsu thresholding [48] algorithm is applied to classify the images into two types, changed and unchanged, and then the corresponding binary change images are generated. Sample selection is conducted based on the results of multi-scale segmentation 
and pixel-level pre-classification. For the $i$ th object $R_{i}$, the uncertainty index $T$ is calculated based on pixel-level pre-classification results, as shown in the following Equation (6):

$$
T= \begin{cases}\frac{n_{\mathrm{c}}}{n} & n_{\mathrm{c}} \geq n_{\mathrm{u}} \\ -\frac{n_{\mathrm{u}}}{n} & n_{\mathrm{c}}<n_{\mathrm{u}}\end{cases}
$$

where $n_{\mathrm{c}}, n_{\mathrm{u}}$, and $n$ are the numbers of changed, unchanged, and total pixels in object $R_{i}$, respectively. After setting the threshold $T_{\mathrm{m}}$, we use the following equation to determine the properties of the object:

$$
l_{i}= \begin{cases}1 & T<-T_{\mathrm{m}} \\ 2 & -T_{\mathrm{m}} \leq T \leq T_{\mathrm{m}} \\ 3 & T>T_{\mathrm{m}}\end{cases}
$$

where $l_{i}=1,2,3$ indicates the attributes of object $R_{i}$ that are unchanged, uncertain, and changed, respectively, and the threshold range is $T_{\mathrm{m}} \in(0.5,1)$. Changed and unchanged objects are selected as training samples for RoF, and uncertain objects are further classified.

\subsection{Multi-Feature Information Extraction}

After sample selection, the key steps are feature extraction and analyzes. Due to the characteristics of high-resolution images, comprehensive consideration of ground objects is necessary, which is achieved by combining multiple features. It is necessary to preliminarily specify the features to be extracted according to the requirements of CD [49]. Prior to feature analyses, we filter or combine the extracted features. Finally, based on these features, change information is extracted. After fine multi-scale segmentation, eCognition can determine the features of objects by evaluating the image objects as well as their embedding in the image object hierarchy. In this paper, spectral features and texture features of objects at the same position are extracted from T1 and T2 phase images, respectively.

Spectral features include the mean value, standard deviation, ratio (i.e., the amount that a given image layer contributes to the total brightness), maximum value (i.e., the value of the pixel with the maximum layer intensity value of the image object), and minimum value (i.e., the value of the pixel with the minimum layer intensity value in the image object). Haralick et al. [50] used the Grey-level Co-occurrence Matrix (GLCM) method to define 14 types of texture features. In this paper, the primary texture features are the mean value, standard deviation, contrast, entropy, homogeneity, correlation, angular second moment, and dissimilarity. The spectral and texture features are stacked, as shown in Figure 4. Table 1 lists the features extracted in this study. Each type of feature may reflect different object information from different angles, and often complement each other. After extracting the two types of features listed above, all features are normalised to the range of 0-1 [49] and combined as input RoF data for model training.

Table 1. Overview of extracted features.

\begin{tabular}{ccc}
\hline Object Features & Feature Dimension & Tested Features ( $N$ Bands) \\
\hline Spectral features & $10 \times N$ & Mean value, standard deviation, ratio, maximum value, \\
minimum value
\end{tabular}




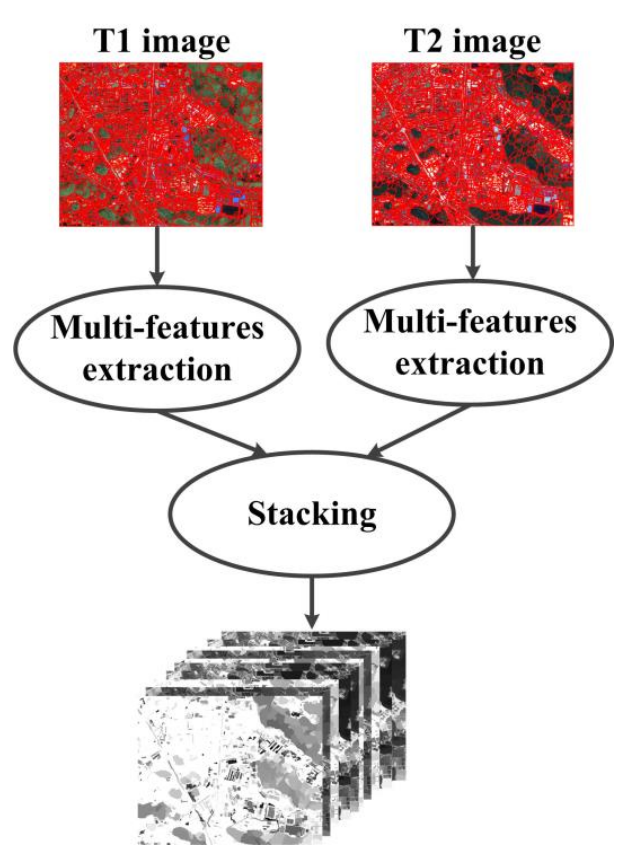

Figure 4. Multi-feature stacking scheme.

\subsection{OBCD Based on RoF and Coarse-to-Fine Uncertainty Analyses}

For multi-scale image sequences, the coarse-scale object size is large, which is suitable for CD of objects with large areas. The fine-scale object size is small, and it is advantageous for CD of small objects. Therefore, detecting large objects at a coarse scale, detecting small objects at a fine scale, and then synthesising the object detection results at different scales is useful for improving the accuracy and reliability of the $\mathrm{CD}$ algorithm. Inspired by the concept of "from coarse to fine, refine layer by layer" [25], this paper proposes a coarse-to-fine uncertainty analysis method. The overall workflow is shown in Figure 5, which includes the following general steps:

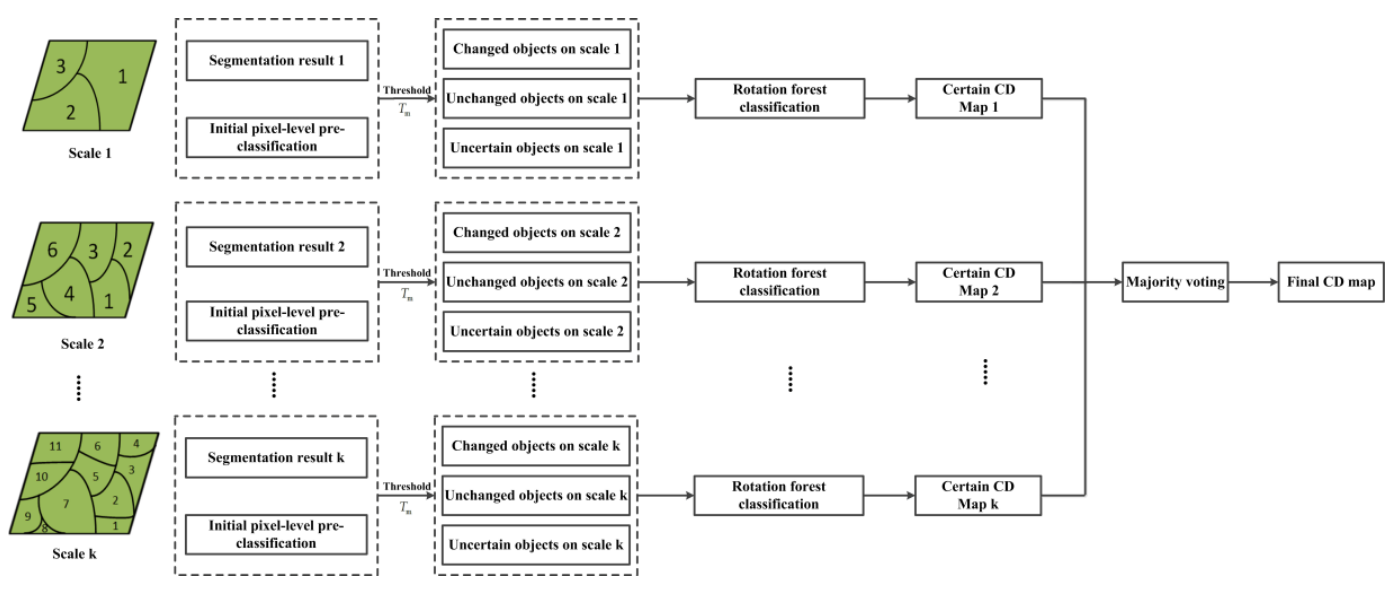

Figure 5. OBCD process incorporating rotation forest and coarse-to-fine uncertainty analyses.

\section{Step 1: Uncertain object classification by RoF}

The RoF classifier method can successfully generate classifier ensembles based on feature extraction. This paper utilises RoF to randomly divide the original feature dataset into several subsets, and carries out feature transformation (e.g., principal component analysis, PCA) for each subset $[23,36-38,51]$. The correlations between transformed subsets are minimized. This work lays the foundation for 
improving the accuracy of classification without changing the information in the original dataset by retaining all major components. The classification and regression tree (CART) [23] is employed as the base classifier because it is sensitive to rotation of the characteristic axis and can produce a strongly differentiating classifier. The decision tree is intuitive and easy to understand. The training of each base classifier is carried out in different subsets, significantly increasing the difference with the base classifier. This training helps to improve the accuracy of prediction.

Step 2: Coarse-to-fine image fusion

The proposed approach makes full use of the advantages of multi-scale image analyses and expression, and simultaneously uses multi-scale image layer object relationships to fuse coarse-scale CD results with fine-scale results. Finally, the MV rule [23] is used to obtain the final $\mathrm{CD}$ results.

\section{Experiments and Results}

\subsection{Dataset Description}

To verify the feasibility and effectiveness of the proposed method, we apply the proposed method to two pairs of real high-resolution remote sensing datasets. The first experimental dataset (DS1) contains GF2 multi-spectral images (Figure 6a,b) captured in 2015 and 2016 covering the city of Liuzhou, China. GF2 is a Chinese satellite launched in August 2014, which provides high-resolution imagery of the Earth. The orbit height of the GF2 satellite is $631 \mathrm{~km}$, and its inclination is $97.9080^{\circ}$. The GF2 image comprises four spectral bands: Red (R), green (G), blue (B), and near infrared, as well as one panchromatic band. The pan- and multi-spectral images are fused using the Pan-sharp algorithm [52] and the spatial resolution of the fused image is $0.8 \mathrm{~m}$. The bi-temporal images used for experiments are orthorectified and mainly include the three bands R, G, and B. The image contains $3749 \times 3008$ pixels. The vector data used in this study are an HVM of Liuzhou compiled in 2015 (Figure 6c). These data were produced through the National Census Geographic of China. The HVM and images of the same area are obtained after preprocessing. The vector data are projected using Transverse Mercator projection, and the projection's central meridian is $111^{\circ} \mathrm{E}$. The vector data contains 554 objects.

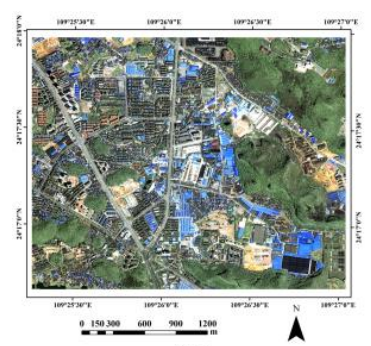

(a)

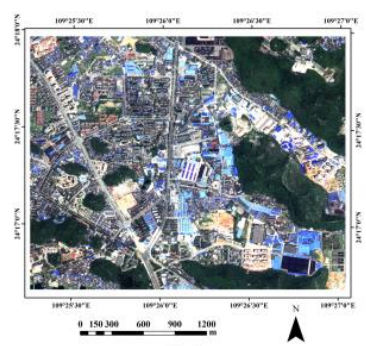

(b)

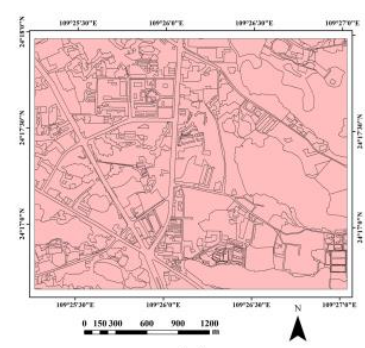

(c)

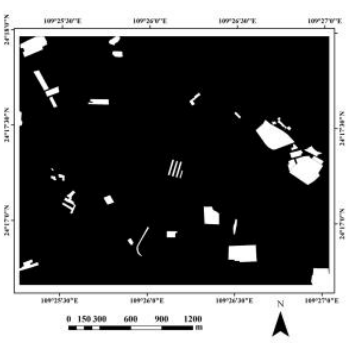

(d)

Figure 6. The first experimental dataset (DS1). (a) Image acquired on 13 April 2015; (b) image acquired on 11 May 2016; (c) HVM compiled in 2015; (d) reference image.

The two high-resolution images in the second dataset (DS2) are also GF2 multi-spectral images (Figure 7a,b). The bi-temporal images used for experiments mainly utilize the three bands of R, G, and $\mathrm{B}$, and contain $5314 \times 4745$ pixels. The image areas are much larger than those of DS1 and include a variety of land cover types. The vector data set for land use contains 1540 objects (Figure 7c). From the bi-temporal images and HVMs, the main land cover types are divided into nine categories, i.e., farmland, woodland, garden land, grassland, building, road, bare land, water and artificial structure. The types are in accordance with the classification criteria of the National Census Geography of China. Among them, artificial structure mainly includes playground, impervious surface and construction; building is categorized into medium-story apartments, commercial building, industrial building, 
settlement and residential area; grassland is categorized into artificial grassland and natural grassland; farmland is categorized into paddy field and dry land; garden land is categorized into orchard, tea plantation and mulberry field. The reference images shown in Figures $6 \mathrm{~d}$ and $7 \mathrm{~d}$ were produced using the polygon to raster (PTR) tool built into ESRI ArcGIS 10.1 software. Reference polygon features were produced through the National Census Geography of China. The black areas represent unchanged regions, while white regions have changed.

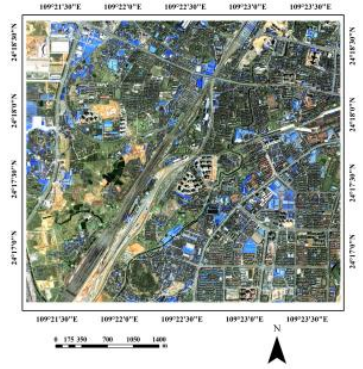

(a)

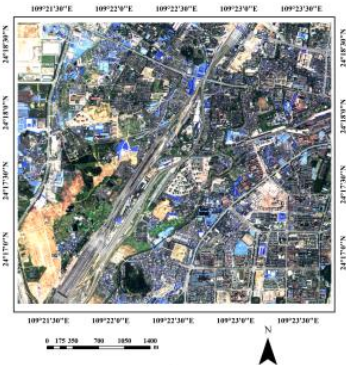

(b)

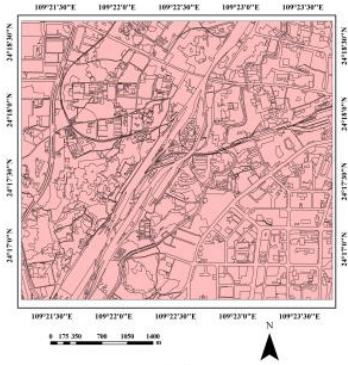

(c)

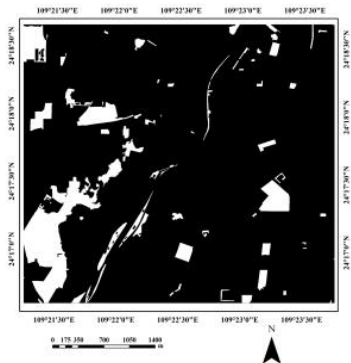

(d)

Figure 7. The second experimental dataset (DS2). (a) Image acquired on 13 April 2015; (b) image acquired on 11 May 2016; (c) HVM in 2015; (d) reference image.

\subsection{Evaluation Metrics}

Evaluation of accuracy is essential to interpreting $\mathrm{CD}$ results and the final decision-making process. Four indices are used to evaluate the accuracy of the final results [53]:

- False alarms (FA): The number of unchanged pixels that are incorrectly detected as having changed, $N_{\mathrm{FA}}$; the false alarm rate is defined as $R_{\mathrm{FA}}=N_{\mathrm{FA}} / N_{0} \times 100 \%$, where $N_{0}$ is the total number of unchanged pixels.

- Missed alarms (MA): The number of changed pixels that are incorrectly detected as being unchanged, $N_{\mathrm{MA}}$; the missed alarm rate is defined as $R_{\mathrm{MA}}=N_{\mathrm{MA}} / N_{1} \times 100 \%$, where $N_{1}$ is the total number of changed pixels.

- Overall error (OE): The total errors caused by FA and MA; the overall alarm rate is calculated as $R_{\mathrm{OE}}=\left(N_{\mathrm{FA}}+N_{\mathrm{MA}}\right) /\left(N_{0}+N_{1}\right) \times 100 \%$.

- Kappa: The Kappa coefficient is a statistical measure of accuracy or agreement, which reflects the consistency between experimental results and ground truth data, and is expressed as Kappa $=\left(p_{0}-p_{\mathrm{c}}\right) /\left(1-p_{\mathrm{c}}\right)$, where $p_{0}$ indicates the true consistency and $p_{\mathrm{c}}$ indicates the theoretical consistency.

\subsection{Experimental Results and Analysis}

To verify the feasibility and effectiveness of the proposed approach, two unsupervised pixel-based CD methods, i.e., PCA- $k$-means [10] and NCIA, as well as three OB methods are used for comparison. The PCA- $k$-means method has two parameters, i.e., non-overlapping blocks $H(H=5$ in our experiments) and the dimensions $S$ ( $S=5$ in our experiments) of the eigenvector space. OCVA [18] is employed as the unsupervised method, and extreme learning machine (ELM) [54] and random forest (RF) [55] are used as the supervised classifiers. The parameters involved in these methods are set as in the original paper. The implementation of the ELM method is available at Ref. 54. These methods are applied to demonstrate the advantages of the proposed approach. The CART decision tree is adopted as the base classifier for RF and RoF. The default number of trees for RF is set to 100, while the number of decision trees for RoF is set to $L=50$. For RoF, Xia et al. [23,38,51] suggest that a small number of features per subset will increase the classification performance, as such, we set $M=10$. The threshold of the uncertainty index is set to $T_{\mathrm{m}}=0.55$ for both experiments after the comparisons. 


\subsubsection{Test of Scale Parameters}

Multi-scalar image segmentation is a fundamental step in OBCD. When using the ESP tool in DS1, the scale range is from 55-245 in steps of 5. The resulting LV and ROC-LV values vary with scale. As illustrated in Figure 8a, as the segmentation scale increases, LV values retain a trend of growth, while ROC-LV values show the opposite trend. The change in LV from one level to another indicates how important that scale level is for structuring information about object variability relative to the whole image. Theoretically, the peaks in an ROC-LV curve indicate the levels where LV increases as segments delineate their correspondents in the real world [42]. In Figure 8a, the peaks in the ROC-LV curve corresponding to scale levels of 105, 115, 125, 150, 175, 210 and potentially 240 indicate the meaningful scale parameters for segmentation of DS1. We selected the most obvious peaks, which dominate their neighborhood, as indicators for optimal scale parameters. Such a scale is generally considered an alternative optimal scale. Based on segmentation results, industrial buildings, settlements and residential areas are better segmented at a scale of 105 than at other scales. Features such as playground, impervious surfaces, construction, and bare land are better segmented at a scale of 175, while woodlands and road areas are better segmented at 210. The object levels delineated with these scale parameters matched the structures in real world for the DS1. These three scales are considered suitable for rough image segmentation. Then these three scales (with floating of \pm 5 ) are used to set up the corresponding ranges of 100-110, 170-180, and 205-215. This paper uses the modified ASEI to further determine the specific optimal scale within each range (Figure 9). The maximum values shown in Figure 9 are 102, 179, and 213 in panels a-c, respectively, which can be considered the optimal segmentation scales of DS1. The mean object value maps at these three optimal scales are shown in Figure 10.

For DS2, the scale range is from 105 to 295 in steps of 5 . The maximum ROC-LV values are obtained at scales of 110, 140, 155, 165, 205, 245, and 275, as shown in Figure 8b. These values represent the meaningful scale parameters and are generally considered an alternative optimal scale for the segmentation of DS2. Based on the segmentation results, commercial buildings, industrial buildings, settlements, and residential areas are segmented better at the scale of 140 than at other scales. On the other hand, playgrounds, impervious surfaces, construction areas, and bare land are better segmented at a scale of 205, while grasslands and roads are better segmented at 245. These three scales (with floating of \pm 5 ) are used to set the three corresponding ranges 135-145, 200-210, and 240-250. Then the modified ASEI is used to determine the specific optimal scale for each range (Figure 11). The maximum values in Figure 11 are 136, 206, and 244 in panels a-c, respectively, which can be considered the optimal segmentation scales of DS2. The mean object value maps at these three optimal scales are shown in Figure 12.

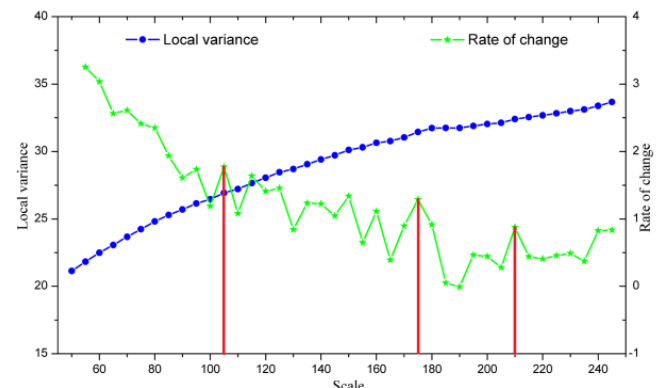

(a)

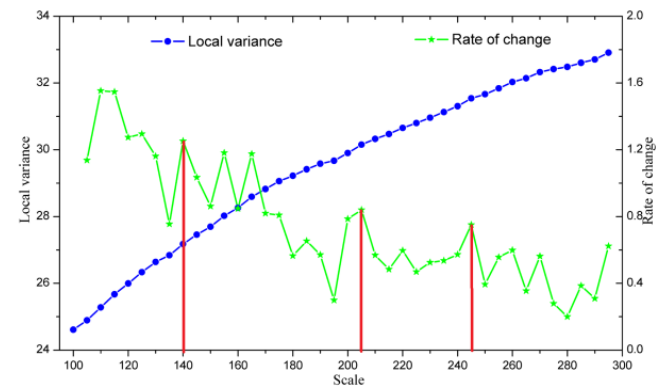

(b)

Figure 8. Curves showing that LV and ROC-LV vary as functions of scale. (a) DS1; (b) DS2. 


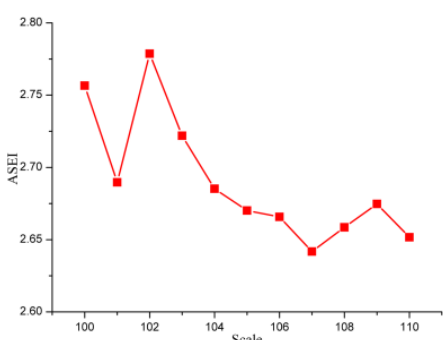

(a)

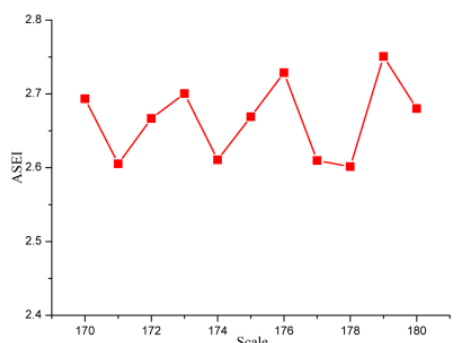

(b)

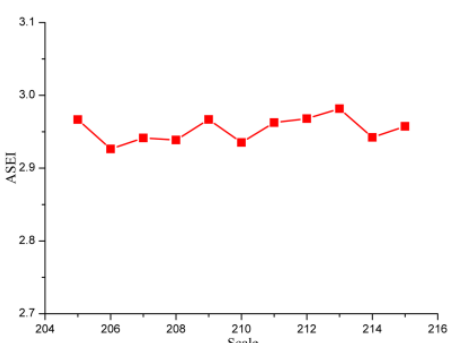

(c)

Figure 9. Line chart of the ASEI index for DS1 at scale ranges of (a) 100 to 110, (b) 170 to 180, and (c) 205 to 215 .

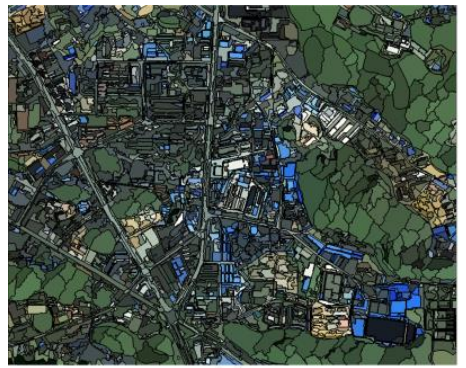

(a)

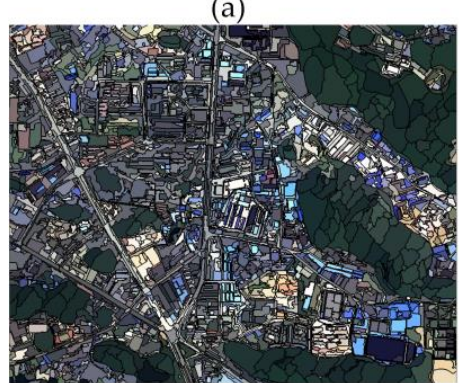

(d)

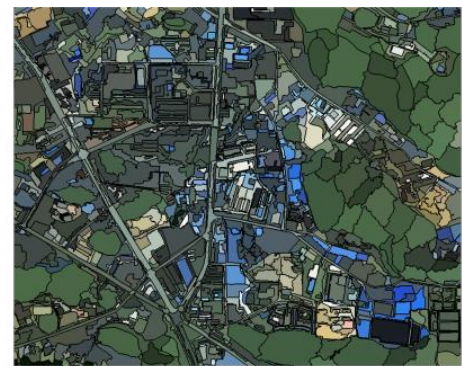

(b)

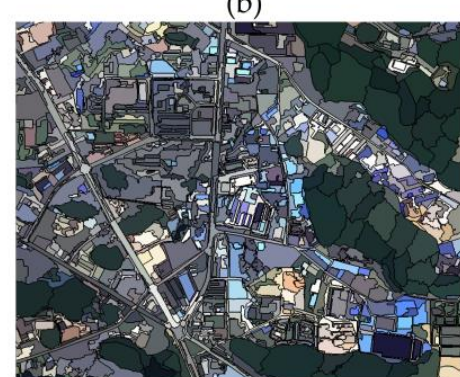

(e)

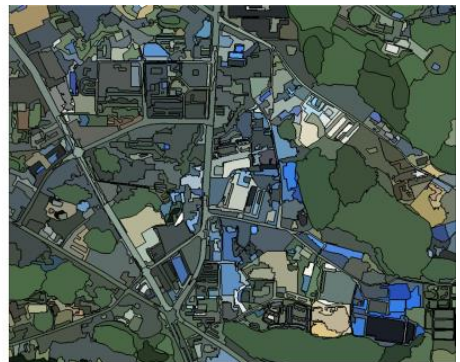

(c)

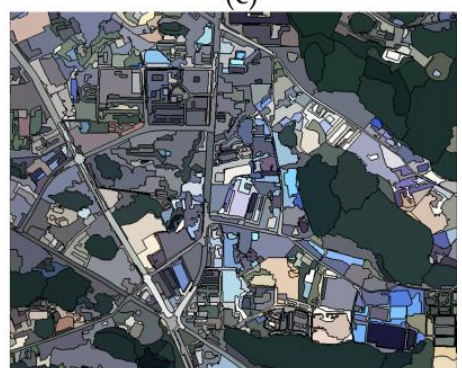

(f)

Figure 10. Mean object value maps for DS1 at various optimal scales. (a-c) Scales of 102, 179, and 213 for the image acquired on 13 April 2015; (d-f) scales of 102, 179, and 213 for the image acquired on 11 May 2016.

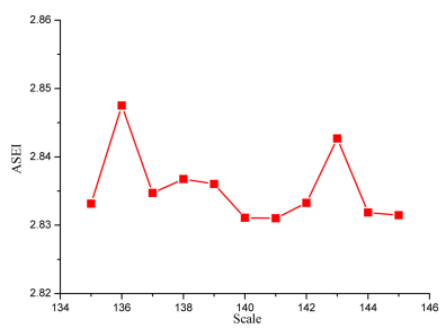

(a)

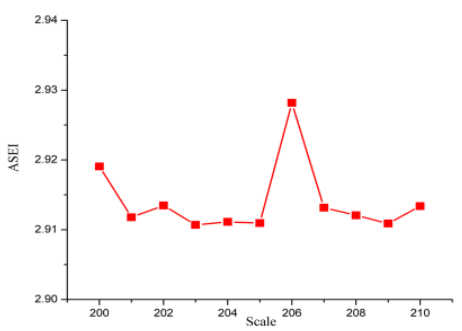

(b)

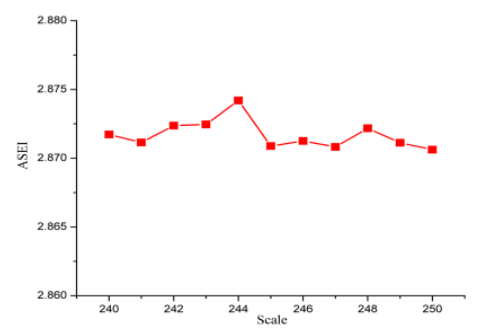

(c)

Figure 11. Line chart of the ASEI index for DS2 at scale ranges of (a) 135 to 145, (b) 200 to 210, and (c) 240 to 250 . 


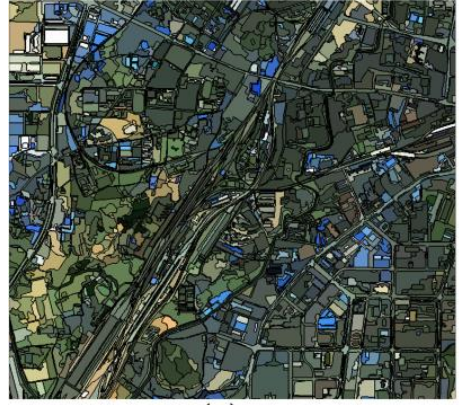

(a)

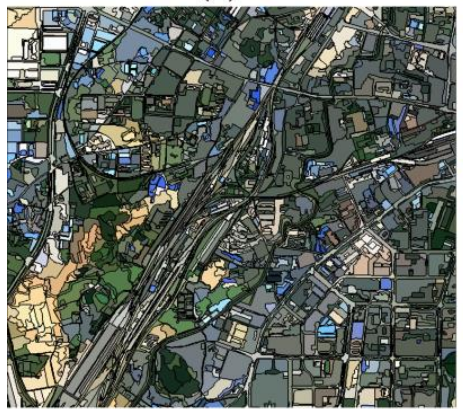

(d)

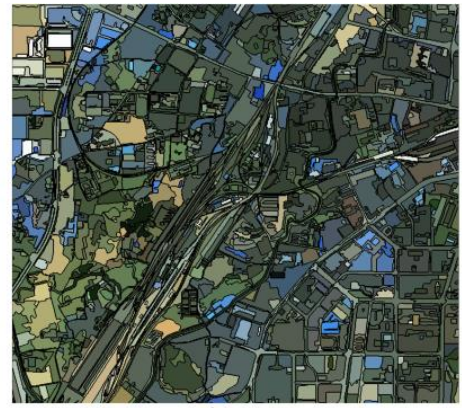

(b)

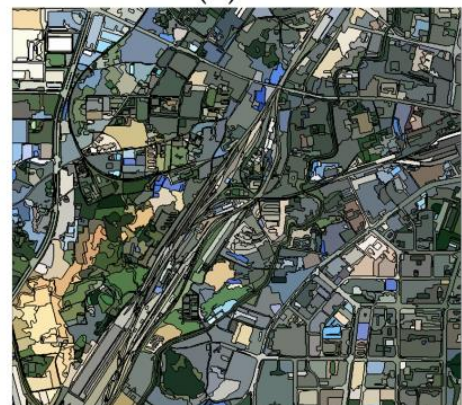

(e)

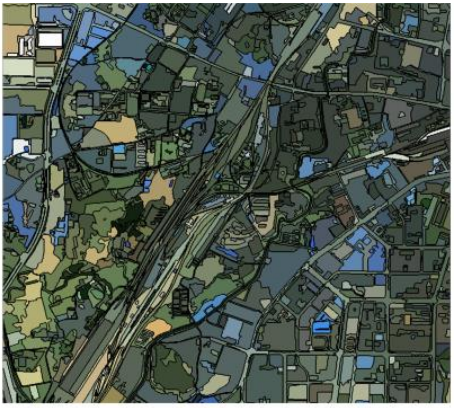

(c)

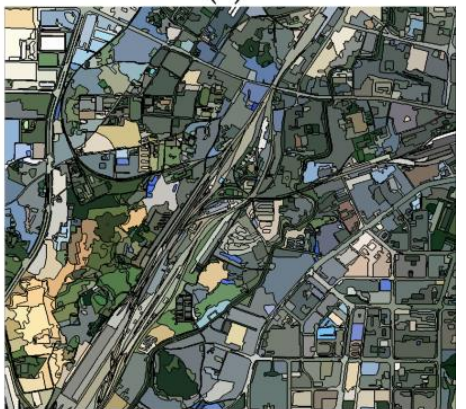

(f)

Figure 12. Mean object value maps for DS1 at different optimal scales. (a-c) Scales of 136, 206, and 244 for the image acquired on 13 April 2015; (d-f) Scales of 136, 206, and 244 for the image acquired on 11 May 2016.

\subsubsection{Results for DS1}

Our results are demonstrated in two ways. The $\mathrm{CD}$ results are displayed in figures and the criteria are listed in tables. Figures 13 and 14 show the experimental results based on DS1. Figure 13 shows $\mathrm{CD}$ results at the three optimal scales using RoF. The false detection phenomenon (the red regions) is most serious at scale 102, whereas the missed detection (the yellow regions) is better at this scale than at the other two scales (scale 179 and scale 213) tested. As the scale increases, false detection is further reduced at scale 213 , but missed detection is slightly more frequent than at the other two scales. This paper utilizes the MV approach to fuse the CD results at the three optimal scales, which can effectively integrate and complement false detection or missed detection phenomena at a single scale and improve accuracy of the final result. Figure 14 shows the results of MV using ELM, RF and RoF. Changed regions are concentrated mainly within strongly changed regions (change occurs at three scales) and obviously changed regions (change occurs at any two scales), while subtly changed regions (change occurs only at one scale) are mostly false changes.

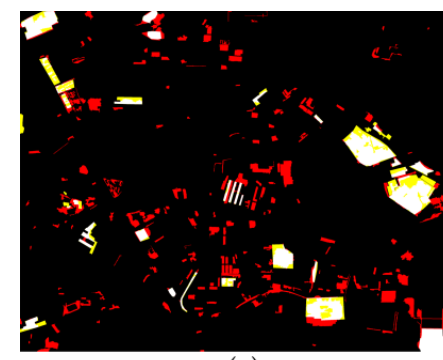

(a)
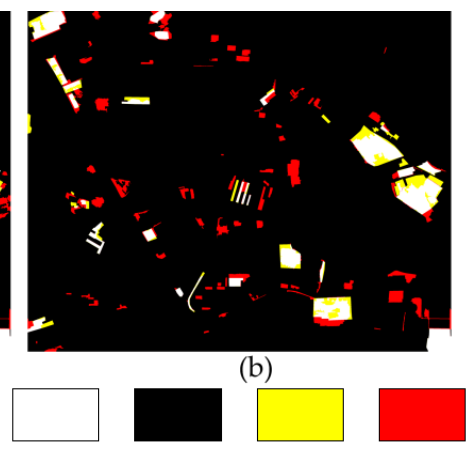

Change

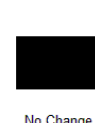

No Change

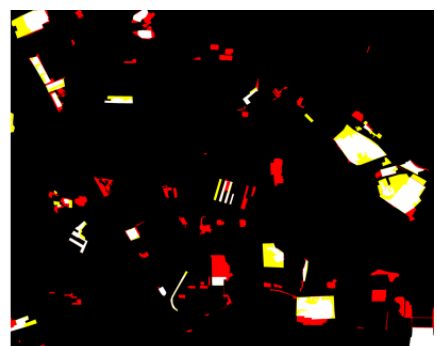

(c)

Figure 13. CD results of DS1. (a) RoF (Scale 102); (b) RoF (Scale 179); (c) RoF (Scale 213). 


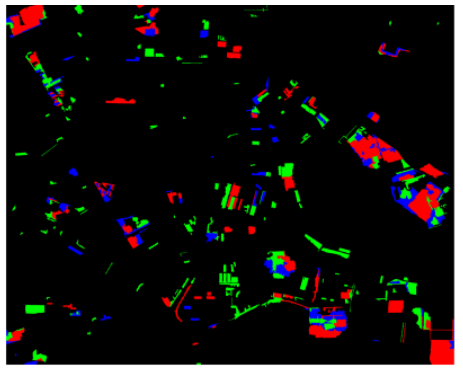

(a)

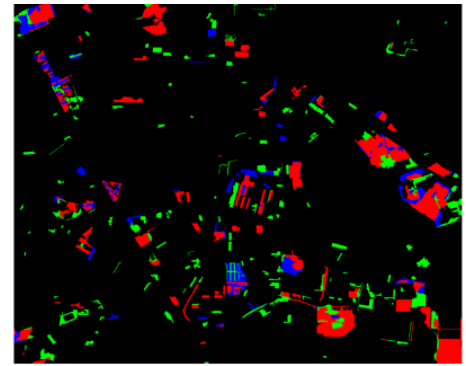

(b)

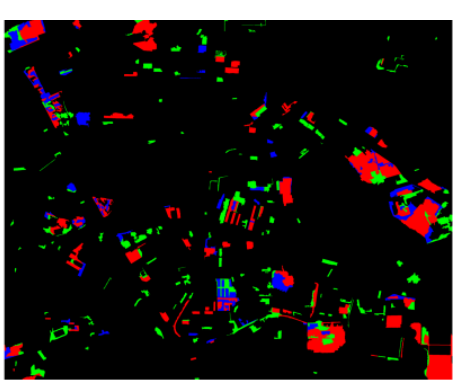

(c)

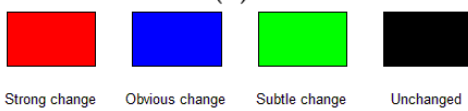

Figure 14. CD results based on majority voting. (a) ELM-MV; (b) RF-MV; (c) RoF-MV.

The proposed fusion strategy converts the traditional "hard detection" method into "soft detection". Various scale detection results are combined to attain stepwise reclassification of the change intensity, which offers a more meaningful and flexible reference in practice than simply separating all pixels into two classes of changed and unchanged. Moreover, strong changes that occur at all three scales can be defined as the areas with maximum probability of change, while obvious changes that occur at two scales are viewed as having the second greatest probability. Finally, the strongly and obviously changed regions are considered changed areas, whereas the subtly changed and unchanged regions are treated as unchanged areas.

Table 2 lists the values used for evaluation metrics. As shown in Figure 15, the two pixel-based methods are filled many white spots due to noise. The CD accuracy of pixel-based methods is comparatively low, mainly because only the spectral features of bi-temporal images are used in these methods. Furthermore, due to the high resolution of the original images, pixel-based CD methods based on spectral statistics are not able to meet the requirements for change information extraction and the results obtained are poor. The OCVA method achieves better object-level CD results than the two pixel-based methods. Because the unsupervised CD method is strongly affected by the threshold, OCVA results are worse than those of the supervised RoF at the three optimal scales. The dominant change combinations include those between settlements and impervious surfaces, and between bare land and buildings. The main driver of these changes is urban growth and construction in Lizhou in recent years. Due to the limitations of OCVA, some changed objects are falsely detected as unchanged, while some unchanged objects are falsely identified as changed. The scale constraints are fully considered through implementation of coarse-to-fine uncertainty analyses. The amounts of uncertain data are reduced with each scale. CD results at the three optimal scales are combined to generate final results using the MV approach. Compared to the RF-MV and ELM-MV methods, the kappa coefficient of RoF-MV is highest among the three supervised classifiers. Furthermore, it should be noted that although image segmentation may impair results near borders, the proposed approach not only improves CD performance but also enhances the image boundary compared to results at a single scale. In short, the proposed approach obtains the best results and greater accuracy compared to other methods tested. 

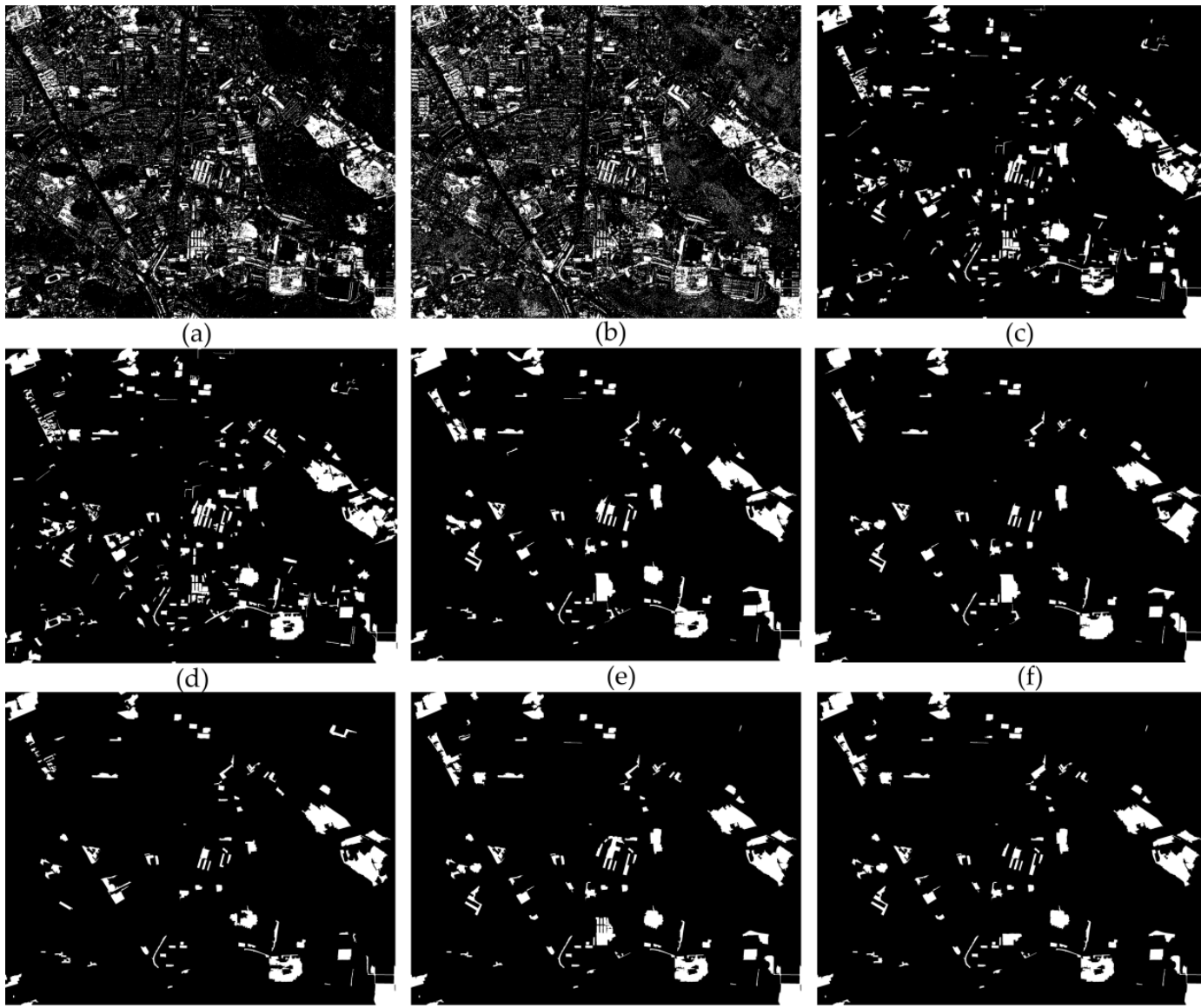

(g)

(h)

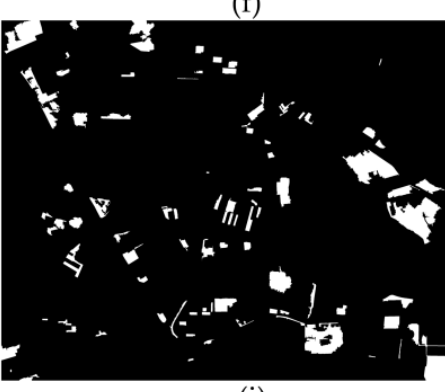

(i)

Figure 15. Comparison of different methods for DS1. (a) NCIA; (b) PCA- $k$-means; (c) OCVA; (d) RoF-scale 102; (e) RoF-scale 179; (f) RoF-scale 213; (g) ELM-MV; (h) RF-MV; (i) RoF-MV.

Table 2. Performance comparison of different approaches for DS1.

\begin{tabular}{|c|c|c|c|c|c|c|c|c|c|}
\hline \multirow[t]{3}{*}{ Method } & \multicolumn{2}{|c|}{ Pixel-Based } & \multicolumn{7}{|c|}{ Object-Based } \\
\hline & \multirow{2}{*}{ NCIA } & \multirow{2}{*}{ PCA- $k$-Means } & \multirow{2}{*}{ OCVA } & \multicolumn{3}{|c|}{ RoF } & \multirow{2}{*}{$\begin{array}{l}\text { RoF } \\
\text { MV }\end{array}$} & \multirow{2}{*}{$\begin{array}{c}\text { RF } \\
\text { MV }\end{array}$} & \multirow{2}{*}{$\begin{array}{c}\text { ELM } \\
\text { MV }\end{array}$} \\
\hline & & & & Scale 102 & Scale 179 & Scale 213 & & & \\
\hline FA $(\%)$ & 12.27 & 16.01 & 4.43 & 4.82 & 3.81 & 2.99 & 3.01 & 3.21 & 2.37 \\
\hline MA (\%) & 34.93 & 36.77 & 31.77 & 28.25 & 23.11 & 31.75 & 26.55 & 26.74 & 34.84 \\
\hline OE (\%) & 13.40 & 17.04 & 5.79 & 5.99 & 4.57 & 4.53 & 4.18 & 4.37 & 3.99 \\
\hline Kappa (\%) & 27.17 & 20.78 & 51.07 & 51.41 & 59.28 & 58.82 & 61.49 & 60.27 & 59.86 \\
\hline
\end{tabular}

\subsubsection{Results for DS2}

Figures 16 and 17 show the experimental results obtained for DS2. As shown in Figure 16, among the three optimal scales, the false detection phenomenon (the red regions) is more serious at scale 136 than at the other two scales (scale 206 and scale 244); on the other hand, the missed detection phenomenon (the yellow regions) is better at that scale. As the scale increases, false detection is further reduced at the scale of 244, but missed detection is slightly higher than at the other two scales. Therefore, these three scales of DS2 can compensate for each other in the CD process. Figure 17 shows the results of ELM, RF, and RoF using the MV approach. Strong and obvious changes can be set as the areas with a maximum probability of change, while subtle changes are viewed as having secondary importance. 


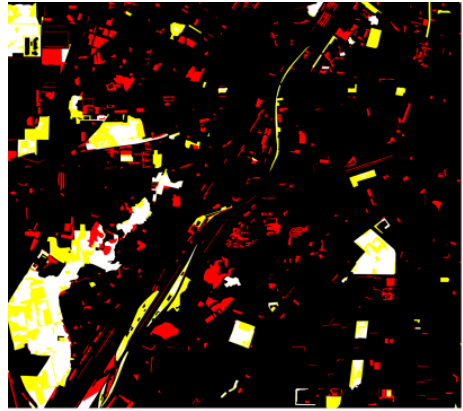

(a)

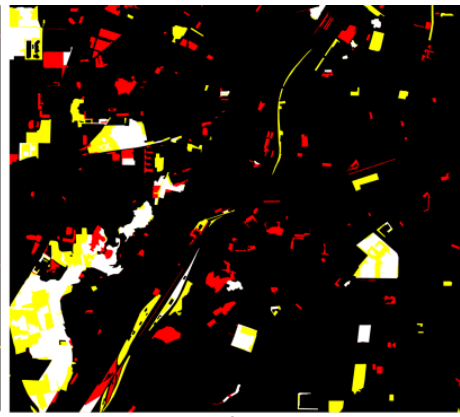

(b)

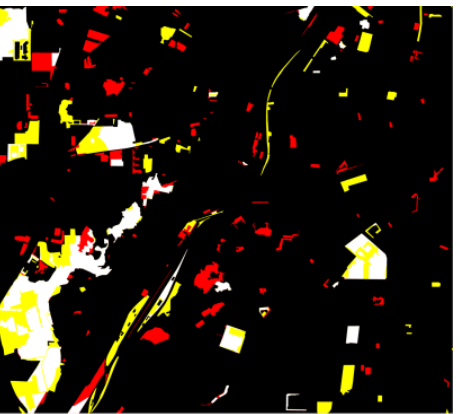

(c)

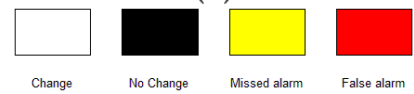

Figure 16. CD results for DS2. (a) RoF (Scale 136); (b) RoF (Scale 206); (c) RoF (Scale 244).

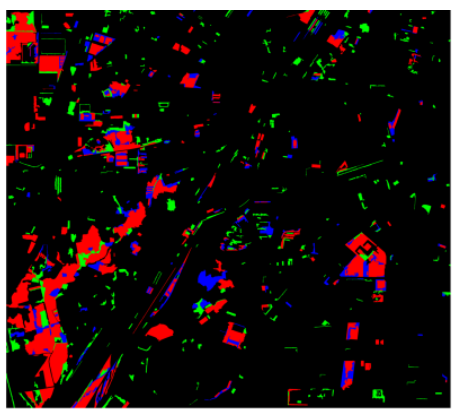

(a)

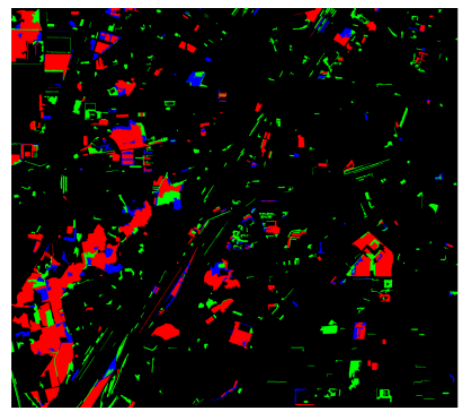

(b)

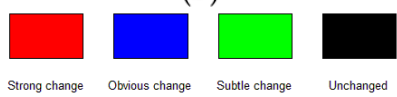

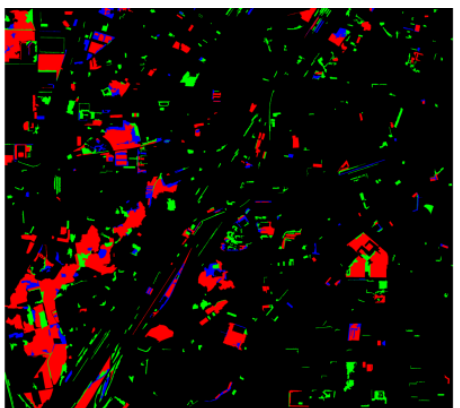

(c)

Figure 17. CD results obtained by majority voting. (a) ELM-MV; (b) RF-MV; (c) RoF-MV.

Four indicators, namely, FA, MA, OE, and the kappa coefficient, are adopted for quantitative comparisons (Table 3). As shown in Figure 18, the results of OBCD methods (OCVA, ELM, RF, and RoF) indicated better performance than those of PBCD methods (NCIA and PCA- $k$-means). The NCIA method utilizes contextual information to make a final decision, and thus performs better than the PCA- $k$-means method. The dominant land cover change combinations indicate changes between roads and impervious surfaces, bare land and farmland, settlements and impervious surfaces, and bare land and buildings. The unsupervised OCVA method achieved better CD result, due to the use of segmented objects as the basic unit of analysis. With this method, changed objects are more regular. The MV scheme obtains better CD results than a single scale, and leads to much more homogeneous regions shown on the CD maps. Furthermore, the proposed RoF-MV method was superior to all other methods in terms of the kappa coefficient for DS2. Regardless of the computation time, it can be expected that RoF can surpass RF to some extent. The time requirement for ELM is lower than RF and RoF classifiers. However, the final kappa coefficient of ELM is smaller than these two ensemble learning methods. The proposed approach clearly outperforms other comparison methods in both qualitative and quantitative analyses. 


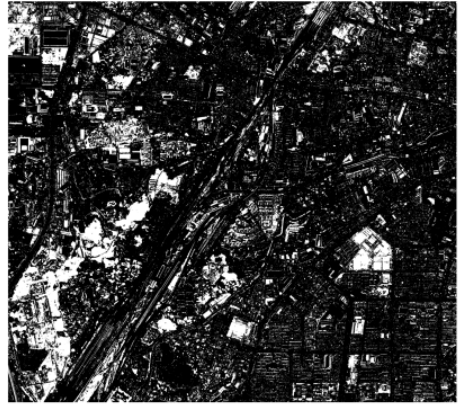

(a)

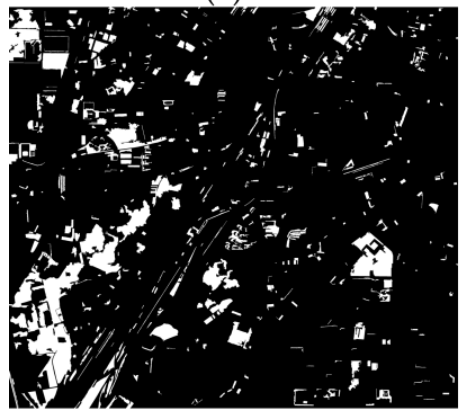

(d)

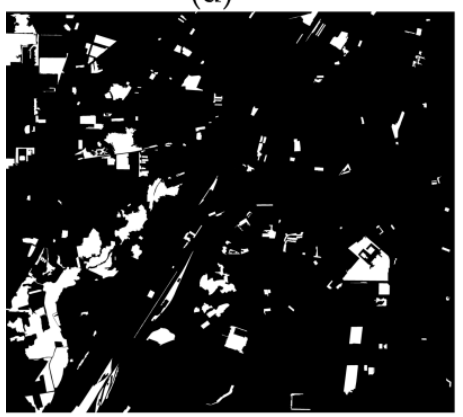

(g)

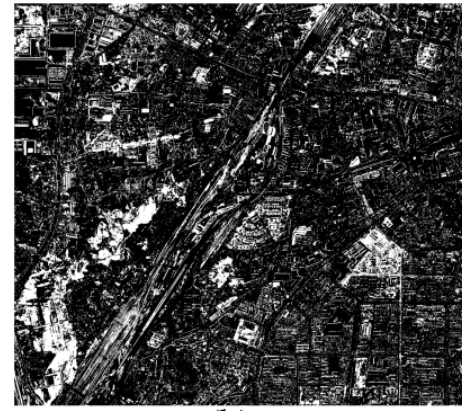

(b)

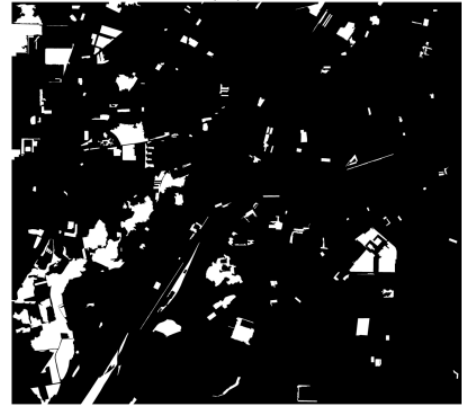

(e)

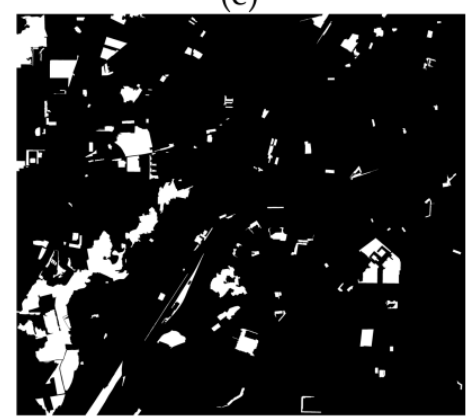

(h)

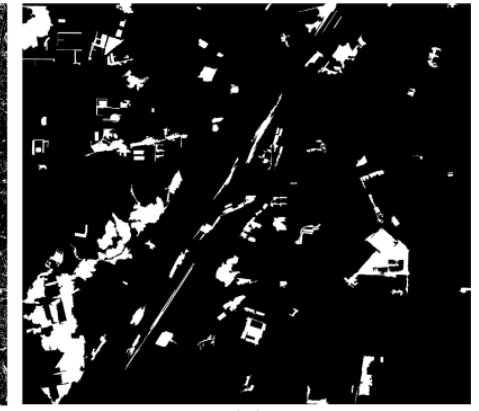

(c)

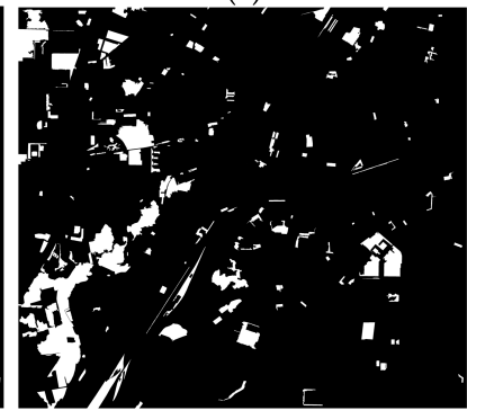

(f)

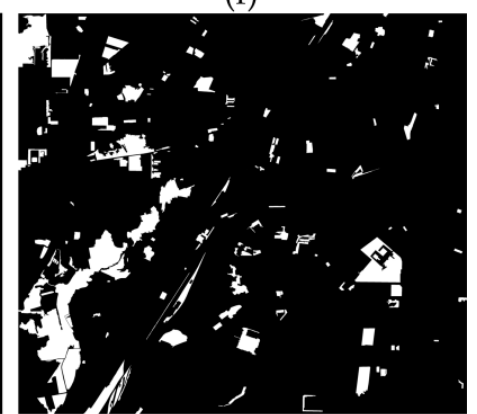

(i)

Figure 18. Comparison of different methods for DS2. (a) NCIA; (b) PCA- $k$-means; (c) OCVA; (d) RoF-scale 136; (e) RoF-scale 206; (f) RoF-scale 244; (g) ELM-MV; (h) RF-MV; (i) RoF-MV.

Table 3. Performance comparison of different approaches for DS2.

\begin{tabular}{|c|c|c|c|c|c|c|c|c|c|}
\hline \multirow{3}{*}{ Accuracy } & \multicolumn{2}{|c|}{ Pixel-Based } & \multicolumn{7}{|c|}{ Object-Based } \\
\hline & \multirow{2}{*}{ NCIA } & \multirow{2}{*}{ PCA- $k$-Means } & \multirow{2}{*}{ OCVA } & \multicolumn{3}{|c|}{ RoF } & \multirow{2}{*}{$\begin{array}{l}\text { RoF } \\
\text { MV }\end{array}$} & \multirow{2}{*}{$\begin{array}{c}\text { RF } \\
\text { MV }\end{array}$} & \multirow{2}{*}{$\begin{array}{c}\text { ELM } \\
\text { MV }\end{array}$} \\
\hline & & & & Scale 136 & Scale 206 & Scale 244 & & & \\
\hline FA $(\%)$ & 13.37 & 17.48 & 4.69 & 5.61 & 3.74 & 3.72 & 3.55 & 3.49 & 4.18 \\
\hline MA (\%) & 42.08 & 52.41 & 53.71 & 43.82 & 47.23 & 46.58 & 42.28 & 45.09 & 44.38 \\
\hline $\mathrm{OE}(\%)$ & 16.22 & 20.95 & 9.57 & 9.41 & 8.07 & 7.98 & 6.67 & 7.63 & 8.17 \\
\hline Kappa (\%) & 32.97 & 20.47 & 43.78 & 49.08 & 52.12 & 52.71 & 56.72 & 54.69 & 52.99 \\
\hline
\end{tabular}

\section{Discussion}

Scale is an important feature in the OBCD process. The accuracy of OBCD results depends greatly on the quality of the MTIS and information extraction methods. Contextual constraints link parent and child objects at various scales. In this paper, highly homogenous objects with consistent spatial positions are obtained using the MRS algorithm under the boundary constraints of the HVM. The proposed approach, which is capable of delineating and analysing image ground objects at different scales based on coarse-to-fine uncertainty analyses, was successfully implemented for high-resolution remotely sensed images. The approach adopts RoF to classify uncertain regions of the generated coarse-to-fine segmentation maps. Multiple CD results are combined to generate the final result 
using the MV approach. Experimental results demonstrated the effectiveness of multi-feature and coarse-to-fine image fusion in improving $\mathrm{CD}$ results. The proposed approach led to acceptable levels of efficiency and accuracy. Furthermore, comparative analyses showed that the accuracy is better than other CD methods. The results support the proposed approach as a highly efficient method to improve monitoring of urban areas using remotely sensed data.

Our approach is based on a combination of pixel-based and OB analyses. The hybrid algorithms that combined pixel- and object-based schemes successfully reduced noisy changes, as well as the small and spurious changes introduced by the inconsistent delineation of objects. However, quality of the training samples is affected by the initial pixel-level pre-classification results. The threshold of uncertainty index $T_{\mathrm{m}}$ is an important parameter that can affect the final $\mathrm{CD}$ results. The performance varies with different threshold values. In this paper, the two experimental datasets dynamically adjust $T_{\mathrm{m}}$ within the interval $(0.5,1)$ at a step size of 0.1. As shown in Figure 19, among the three optimal scales for DS1, the false and overall alarm rates at scale 213 are lower than those of scale 102 and 179 as the threshold $T_{\mathrm{m}}$ value increases, whereas the missed alarm rate shows the opposite trend. Similarly, when the threshold is within the interval $(0.5,0.85)$, the false and overall alarm rates at scale 102 are higher than those at scales of 179 and 213, whereas the missed alarm rate shows the opposite pattern. When threshold $T_{\mathrm{m}}=0.55$, the missed alarm rates at the three optimal scales are lowest, which means that these three scales can compensate for each other in the CD process. Therefore, the false detection or missed detection phenomena at a single scale can be reduced using multi-scale fusion. Figure 20 shows the influence of this index on DS2. The false alarm rate and overall alarm rate at scale 136 are higher than those at scale 206 and 244 as threshold $T_{\mathrm{m}}$ increases, whereas the missed alarm rate has the opposite trend. When the threshold is in the interval $(0.55,0.85)$, the false alarm rate and overall alarm rate at scale 244 are smaller those ones at scales of 136 and 206. When the threshold $T_{\mathrm{m}}=0.55$, the missed alarm rates are lowest at all three scales. Therefore, the optimal threshold value is $T_{\mathrm{m}}=0.55$ for DS2. Using the multi-scale propagation method, incorporation of spatial information at different scales by MRS into the RoF classifier can significantly improve the classifier's performance, which indicates the importance of spatial information. The excellent performance of the proposed approach in our two experiments is mainly attributable to the proposed CD scheme successfully taking advantage of spectral, texture, and spatial information at different scale levels.

In the application of $\mathrm{CD}$, we can combine the pixel-based pre-classification and object-based image analyses approaches for different purposes using RoF and HVM, to obtain final object-level $\mathrm{CD}$ results. The changed objects are more regular, and the object geometries correspond to actual geographical features. Therefore, the combination not only exhibits the advantages of both pixel-based and object-based approaches, but also has the greatest accuracy.

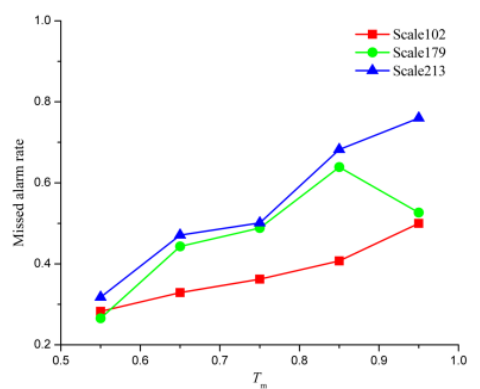

(a)

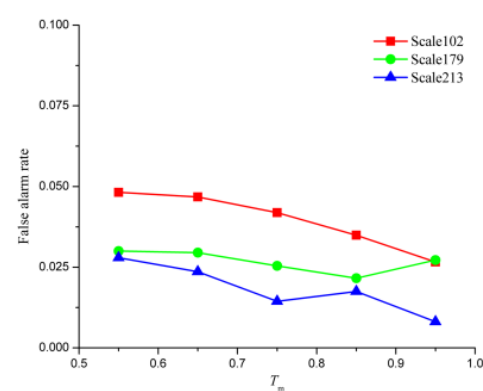

(b)

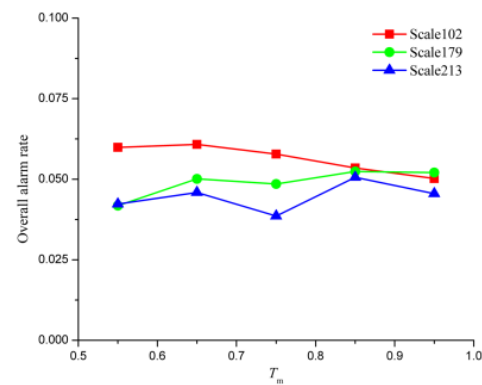

(c)

Figure 19. The influence of the uncertainty index threshold for DS1. (a) Missed alarm rate at various scales; (b) False alarm rate at various scales; (c) Overall alarm rate at various scales. 


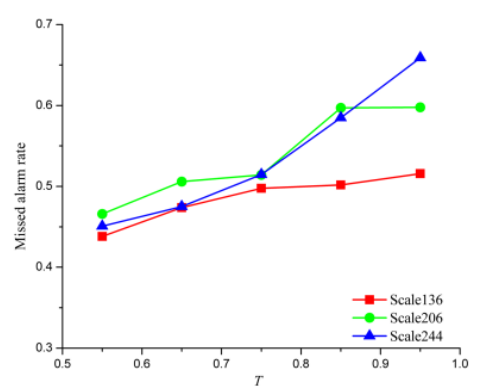

(a)

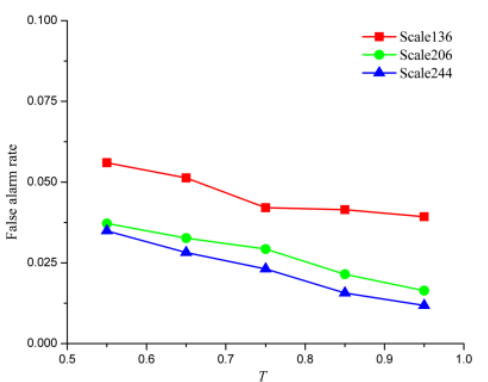

(b)

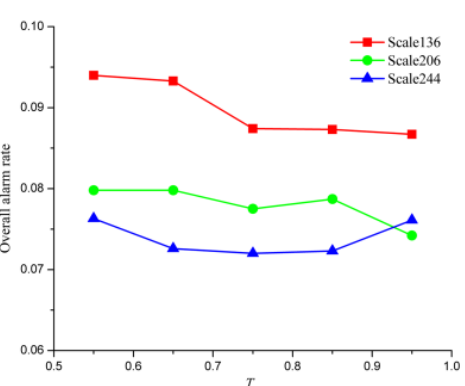

(c)

Figure 20. Influence of the uncertainty index threshold for DS2. (a) Missed alarm rate at various scales; (b) False alarm rate at various scales; (c) Overall alarm rate at various scales.

\section{Conclusions and Perspective}

Optimal scale selection has been a key issue affecting multi-scale segmentation of high-resolution remote sensing images. Determination of the optimal segmentation scale is directly related to subsequent extraction and analysis of change information. In view of this problem, a novel CD approach for high-resolution remote sensing images based on leveraging $\mathrm{RoF}$ and coarse-to-fine uncertainty analyses is proposed in this paper. This approach takes advantage of spatial and contextual information in existing HVM to automatically detect land-use changes. A coarse-to-fine CD strategy is adopted to effectively select local and global changed areas within the HVM.

During the pixel-level pre-classification stage, the NCIA algorithm is used to consider neighborhood information and select pixels with high probabilities of being either changed or unchanged. In the object-level classification stage, a series of optimal segmentation scales ranging from coarse to fine is chosen under the boundary constraints of the HVM. These two methods can effectively avoid subjectivity in scale selection. To obtain more evenly distributed training samples, the proposed sample selection strategy introduces an uncertainty index for each object. The uncertain objects are further classified at a later stage. Multiple classifiers, each of which combines RoF and a single-scale segmentation map, are used to obtain a single-scale classification result. Then, multiple results are integrated using the MV approach to generate the final results. Thus, spatial information from different segmentation scales is incorporated into the $\mathrm{CD}$ process of high-resolution remote sensing images, rather than relying upon a single segmentation scale. Two pairs of real high-resolution remote sensing datasets are used to verify the effectiveness and superior performance of the proposed method. In future research, we will further extend this method from the supervised approach to an unsupervised or semi-supervised approach. In addition, we will focus on automated segmentation and parameter selection processes to further improve performance of the CD.

Author Contributions: This research was designed primarily by W.F. and H.S.; H.S. provided the dataset; W.F., J.T., and K.S. performed the experiments and analyzed the data; W.F. and H.S. wrote the manuscript. W.H. and C.X. reviewed the manuscript and provided theoretical and technical guidance; H.S. acted as the corresponding author.

Acknowledgments: This research was funded by the National Key Research and Development Program of China (No.2016YFB0502603), the National Natural Science Foundation of China under Grant Nos. 41771457, 41471354 and 41601443, and Open Research Fund of the State Key Laboratory of Information Engineering in Surveying, Mapping and Remote Sensing (No.16E01). W. H. was funded by China Scholarship Council and Lund University

Conflicts of Interest: The authors declare no conflict of interest.

\section{Abbreviations}

The following abbreviations are used in this manuscript:

CD Change detection

OBCD Object-based change detection

PBCD Pixel-based change detection 


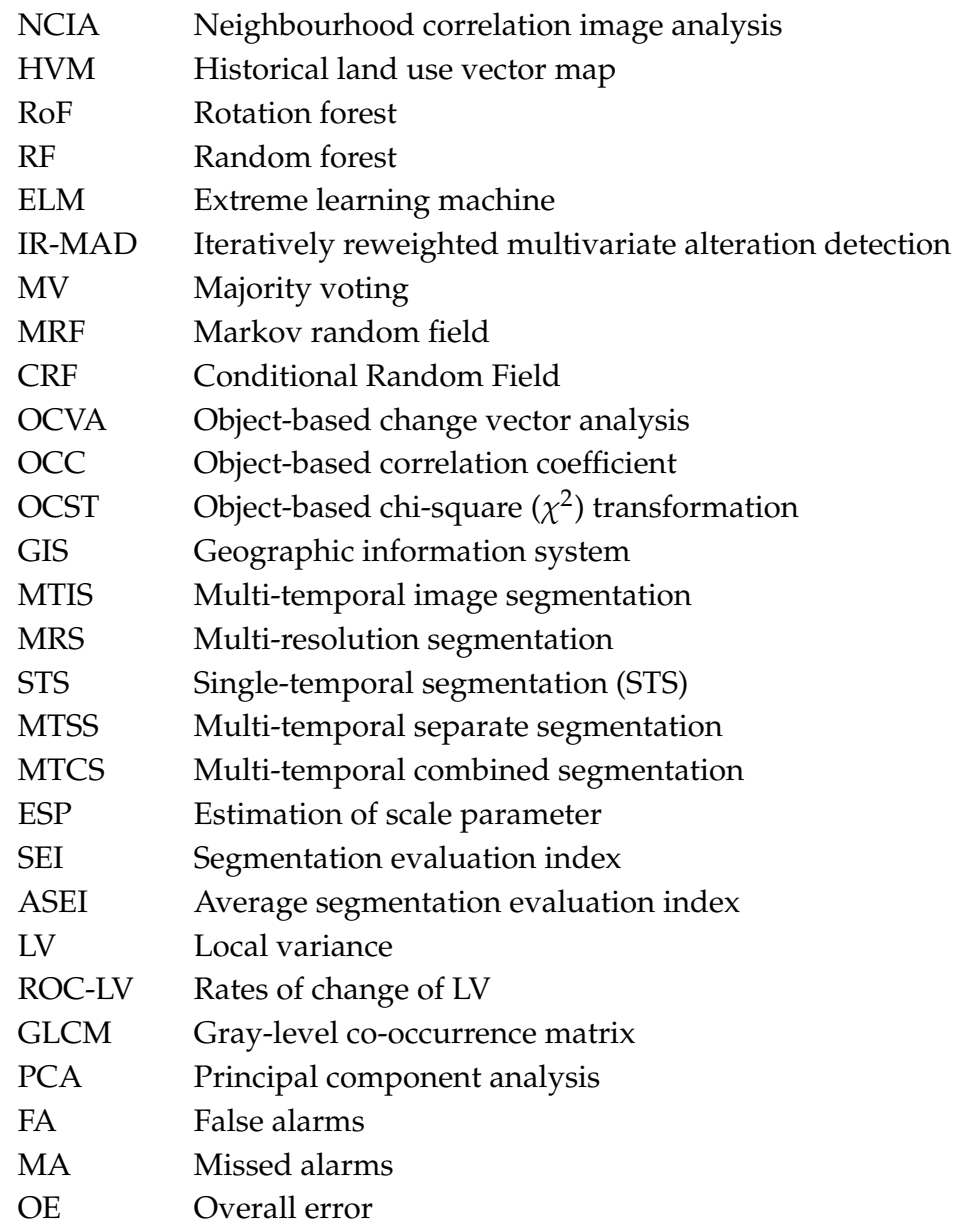

\section{References}

1. Hussain, M.; Chen, D.; Cheng, A.; Wei, H.; Stanley, D. Change detection from remotely sensed images: From pixel-based to object-based approaches. ISPRS J. Photogramm. Remote Sens. 2013, 80, 91-106. [CrossRef]

2. Bovolo, F.; Bruzzone, L. A theoretical framework for unsupervised change detection based on change vector analysis in the polar domain. IEEE Trans. Geosci. Remote Sens. 2007, 45, 218-236. [CrossRef]

3. Hao, M.; Shi, W.; Zhang, H.; Li, C. Unsupervised change detection with expectation-maximization-based level set. IEEE Geosci. Remote Sens. Lett. 2013, 11, 210-214. [CrossRef]

4. Cao, G.; Liu, Y.; Shang, Y. Automatic change detection in remote sensing images using level set method with neighborhood constraints. J. Appl. Remote Sens. 2014, 8. [CrossRef]

5. Bruzzone, L.; Prieto, D. Automatic analysis of the difference image for unsupervised change detection. IEEE Trans. Geosci. Remote Sens. 2000, 38, 1171-1182. [CrossRef]

6. Hao, M.; Zhang, H.; Shi, W.; Deng, K. Unsupervised change detection using fuzzy -means and MRF from remotely sensed images. Remote Sens. Lett. 2013, 4, 1185-1194. [CrossRef]

7. Zhou, L.; Cao, G.; Li, Y.; Shang, Y. Change detection based on conditional random field with region connection constraints in high-resolution remote sensing images. IEEE J. Sel. Top. Appl. Earth Observ. Remote Sens. 2016, 9, 3478-3488. [CrossRef]

8. Cao, G.; Zhou, L.; Li, Y. A new change-detection method in high-resolution remote sensing images based on a conditional random field model. Int. J. Remote Sens. 2016, 37, 1173-1189. [CrossRef]

9. Nielsen, A. The regularized iteratively reweighted mad method for change detection in multi- and hyper-spectral data. IEEE Trans. Image Process. 2007, 16, 463-478. [CrossRef] [PubMed]

10. Celik, T. Unsupervised change detection in satellite images using principal component analysis and $k$-means clustering. IEEE Geosci. Remote Sens. Lett. 2009, 6, 772-776. [CrossRef]

11. Hazel, G. Object-level change detection in spectral imagery. IEEE Trans. Geosci. Remote Sens. 2001, 39, 553-561. [CrossRef] 
12. Wang, W.J.; Zhao, Z.M.; Zhu, H.Q. Object-oriented Change Detection Method Based on Multi-scale and Multi-Feature Fusion. In Proceedings of the 2009 Urban Remote Sensing Joint Event, Shanghai, China, 20-22 May 2009; pp. 1-5.

13. Emary, E.M.; El-Saied, K.M.; Onsi, H.M. A proposed multi-scale approach with automatic scale selection for image change detection. Egypt. J. Remote Sens. Space Sci. 2010, 13, 1-10. [CrossRef]

14. Wang, C.; Xu, M.X.; Wang, X.; Zheng, S.; Ma, Z. Object-oriented change detection approach for high-resolution remote sensing images based on multi-scale fusion. J. Appl. Remote Sens. 2013, 7. [CrossRef]

15. Hao, M.; Shi, W.; Zhang, H.; Wang, Q.; Deng, K. A scale-driven change detection method incorporating uncertainty analysis for remote sensing images. Remote Sens. 2016, 8. [CrossRef]

16. Xiao, P.; Zhang, X.; Wang, D. Change detection of built-up land: A framework of combining pixel-based detection and object-based recognition. ISPRS J. Photogramm. Remote Sens. 2016, 119, 402-414. [CrossRef]

17. Xiao, P.; Yuan, M.; Zhang, X.; Feng, X.; Guo, Y. Cosegmentation for object-based building change detection from high-resolution remotely sensed images. IEEE Trans. Geosci. Remote Sens. 2017, 55, 1587-1603. [CrossRef]

18. Sun, K.; Chen, Y. The Application of objects change vector analysis in object-level change detection. In Proceedings of the International Conference on Computational Intelligence and Industrial Application (PACIIA), Wuhan, China, 6-7 November 2010; Volume 15, pp. 383-389.

19. Wang, L.; Yan, L.I.; Wang, Y. Research on land use change detection based on an object-oriented change vector analysis method. Geogr. Res. 2014, 27, 74-80.

20. Wang, Y.; Shu, N.; Gong, Y. A study of land use change detection based on high resolution remote sensing images. Remote Sens. Land Resour. 2012, 24, 43-47.

21. Chen, Q.; Chen, Y. Multi-Feature Object-Based Change Detection Using Self-Adaptive Weight Change Vector Analysis. Remote Sens. 2016, 8. [CrossRef]

22. Vázquezjiménez, R. Applying the chi-square transformation and automatic secant thresholding to Landsat imagery as unsupervised change detection methods. J. Appl. Remote Sens. 2017, 11. [CrossRef]

23. Chen, J.; Xia, J.; Du, P.; Chanussot, J. Combining Rotation Forest and Multi-scale Segmentation for the Classification of Hyper-spectral Data. IEEE J. Sel. Top. Appl. Earth Observ. Remote Sens. 2016, 9, 4060-4072. [CrossRef]

24. Gang, C.; Geoffrey, J.; Luis, M.; Michael, A. Object-based change detection. Int. J. Remote Sens. 2012, 33, 4434-4457.

25. Zhang, Y.; Peng, D.; Huang, X. Object-Based Change Detection for VHR Images Based on Multiscale Uncertainty Analysis. IEEE Geosci. Remote Sens. Lett. 2018, 15, 13-17. [CrossRef]

26. Alboody, A.; Sedes, F.; Inglada, J. Post-classification and spatial reasoning: New approach to change detection for updating GIS database. In Proceedings of the IEEE International Conference on Information and Communication Technologies: From Theory To Applications, Damascus, Syria, 7-11 April 2008; pp. 1-7.

27. Yu, C.; Shen, S.; Huang, J.; Yi, Y. An object-based change detection approach using high-resolution remote sensing image and GIS data. In Proceedings of the IEEE International Conference on Image Analysis and Signal Processing, Zhejiang, China, 9-11 April 2010; pp. 565-569.

28. Hanif, M.; Mustafa, M.; Hashim, A.; Yusof, K. Spatio-temporal change analysis of Perak river basin using remote sensing and GIS. In Proceedings of the IEEE International Conference on Space Science and Communication, Langkawi, Malaysia, 10-12 August 2015; pp. 225-230.

29. Sofina, N.; Ehlers, M. Building Change Detection Using High Resolution Remotely Sensed Data and GIS. IEEE J. Sel. Top. Appl. Earth Observ. Remote Sens. 2017, 9, 3430-3438. [CrossRef]

30. Ayele, G.T.; Tebeje, A.K.; Demissie, S.S.; Belete, M.A.; Jemberrie, M.A.; Teshome, W.M.; Mengistu, D.T.; Teshale, E.Z. Time Series Land Cover Mapping and Change Detection Analysis Using Geographic Information System and Remote Sensing, Northern Ethiopia. Air Soil Water Res. 2018, 11. [CrossRef]

31. Dewan, A.; Yamaguchi, Y. Land use and land cover change in Greater Dhaka, Bangladesh: Using remote sensing to promote sustainable urbanization. Appl. Geogr. 2009, 29, 390-401. [CrossRef]

32. Wu, C.; Du, B.; Cui, X.; Zhang, L. A post-classification change detection method based on iterative slow feature analysis and Bayesian soft fusion. Remote Sens. Environ. 2017, 199, 241-255. [CrossRef]

33. Aguirre, J.; Seijmonsbergen, A.; Duivenvoorden, J. Optimizing land cover classification accuracy for change detection, a combined pixel-based and object-based approach in a mountainous area in Mexico. Appl. Geogr. 2012, 34, 29-37. [CrossRef] 
34. Lu, J.; Li, J.; Chen, G.; Zhao, L.; Xiong, B.; Kuang, G. Improving Pixel-Based Change Detection Accuracy Using an Object-Based Approach in Multi-temporal SAR Flood Images. IEEE J. Sel. Top. Appl. Earth Observ. Remote Sens. 2015, 8, 3486-3496. [CrossRef]

35. Feng, W.; Sui, H.; Tu, J.; Huang, W.; Sun, K. Remote Sensing Image Change Detection Based on the Combination of Pixel-level and Object-level Analysis. Acta Geod. Cartogr. Sin. 2017, 46, 1147-1155.

36. Lu, X.; Zhang, J.; Li, T.; Zhang, Y. Hyper-spectral Image Classification Based on Semi-Supervised Rotation Forest. Remote Sens. 2017, 9. [CrossRef]

37. Rodríguez, J.; Kuncheva, L.; Alonso, C. Rotation forest: A new classifier ensemble method. IEEE Trans. Pattern Anal. Mach. Intell. 2006, 28, 1619-1630. [CrossRef] [PubMed]

38. Xia, J.; Du, P.; He, X.; Chanussot, J. Hyper-spectral Remote Sensing Image Classification Based on Rotation Forest. IEEE Geosci. Remote Sens. Lett. 2014, 11, 239-243. [CrossRef]

39. Baatz, M.; Schäpe, A. Multi-resolution Segmentation-an optimization approach for high quality multi-scale image segmentation. In Proceedings of the Beiträge zum AGIT-Symposium. 2000, pp. 12-23. Available online: http: / / www.ecognition.com/sites/default/files/405_baatz_fp_12.pdf (accessed on 6 November 2017).

40. Niemeyer, I.; Marpu, P.; Nussbaum, S. Change detection using object features. In Object-Based Image Analysis; Springer: Berlin/Heidelberg, Germany, 2008; pp. 185-201.

41. Zhang, X.; Xiao, P.; Feng, X.; Yuan, M. Separate segmentation of multi-temporal high-resolution remote sensing images for object-based change detection in urban area. Remote Sens. Environ. 2017, 201, 243-255. [CrossRef]

42. Lucian, D.; Dirk, T.; Shaun, R. ESP: A tool to estimate scale parameter for multi-resolution image segmentation of remotely sensed data. Int. J. Geogr. Inf. Sci. 2010, 24, 859-871.

43. Drăguţ, L.; Csillik, O.; Eisank, C.; Tiede, D. Automated parameterisation for multi-scale image segmentation on multiple layers. ISPRS J. Photogramm. Remote Sens. 2014, 88, 119-127. [CrossRef] [PubMed]

44. Chen, C.; Wu, G. Evaluation of optimal segmentation scale with object-oriented method in remote sensing. Remote Sens. Technol. Appl. 2011, 26, 96-102.

45. Liu, H.; Qiu, Z.; Meng, L.; Fu, Q.; Jiang, B.; Yan, Y.; Xu, M. Study on site specific management zone of field scale based on Remote Sensing Image in black soil area. J. Remote Sens. 2017, 21, 470-478.

46. Im, J.; Jensen, J. A change detection model based on neighborhood correlation image analysis and decision tree classification. Remote Sens. Environ. 2005, 99, 326-340. [CrossRef]

47. Im, J. Neighborhood Correlation Image Analysis for Change Detection Using Different Spatial Resolution Imagery. Korean J. Remote Sens. 2006, 22, 337-350.

48. Otsu, N. A threshold selection method from Gray-level. IEEE Trans. Syst. Man Cybern. 1979, 9, $62-66$. [CrossRef]

49. Tan, K.; Jin, X.; Plaza, A.; Wang, X.; Xiao, L.; Du, P. Automatic Change Detection in High-Resolution Remote Sensing Images by Using a Multiple Classifier System and Spectral-Spatial Features. IEEE J. Sel. Top. Appl. Earth Observ. Remote Sens. 2016, 9, 3439-3451. [CrossRef]

50. Haralick, R. Texture features for image classification. IEEE Trans. Syst. Man Cybern. 1973, 3, 610-621. [CrossRef]

51. Xia, J.; Falco, N.; Benediktsson, J.; Chanussot, J.; Du, P. Class-Separation-Based Rotation Forest for Hyperspectral Image Classification. IEEE Geosci. Remote Sens. Lett. 2016, 13, 584-588. [CrossRef]

52. Amro, I.; Mateos, J.; Vega, M.; Molina, R.; Katsaggelos, A. A survey of classical methods and new trends in pansharpening of multispectral images. Eurasip J. Adv. Signal Process. 2016, 9, 3439-3451. [CrossRef]

53. Hou, B.; Wang, Y.; Liu, Q. A Saliency Guided Semi-Supervised Building Change Detection Method for High Resolution Remote Sensing Images. Sensors 2016, 16. [CrossRef] [PubMed]

54. Gao, F.; Dong, J.; Li, B.; Xu, Q.; Xie, C. Change detection from synthetic aperture radar images based on neighborhood-based ratio and extreme learning machine. J. Appl. Remote Sens. 2016, 10. [CrossRef]

55. Wessels, K.; Bergh, F.; Roy, D.; Salmon, B.; Steenkamp, K.; Macalister, B.; Swanepoel, D.; Jewitt, D. Rapid land cover map updates using change detection and robust random forest classifiers. Remote Sens. 2016, 8. [CrossRef]

(C) 2018 by the authors. Licensee MDPI, Basel, Switzerland. This article is an open access article distributed under the terms and conditions of the Creative Commons Attribution (CC BY) license (http://creativecommons.org/licenses/by/4.0/). 